Aus der Abteilung Allgemeinmedizin

(Prof. Dr. med. M. M. Kochen, MPH, FRCGP)

im Zentrum Innere Medizin

der Medizinischen Fakultät der Universität Göttingen

\title{
Compliance bei der hausärztlichen Bluthochdrucktherapie
}

Bewertung eines Instruments zur Selbstbeurteilung von Patienten

\author{
INAUGURAL - DISSERTATION \\ zur Erlangung des Doktorgrades \\ der Medizinischen Fakultät \\ der Georg-August-Universität zu Göttingen \\ vorgelegt von \\ Jörg Schnakenberg \\ aus \\ Bremen
}

Göttingen 2009 
D e k a n: Prof. Dr. med. C. Frömmel

I. Berichterstatter: Prof. Dr. med. M. M. Kochen, MPH, FRCGP

II. Berichterstatter/in:

III. Berichterstatter/in:

Tag der mündlichen Prüfung: 


\section{Inhalt}

$\begin{array}{ll}\text { 1. Einleitung } & 4\end{array}$

2. Stand der Forschung 6

2.1 Der Begriff der Compliance 6

2.2 Die Bedeutung der Compliance in der evidenzbasierten Medizin $\quad 7$

2.3 Die Rolle der Compliance bei der Bluthochdruckbehandlung 9

2.3.1 Prävalenz und Pharmakotherapie des Bluthochdrucks 9

2.3.2 Erhebung der Compliance von Patienten mit Bluthochdruck 10

2.3.3 Erhebung der Compliance nach Hill-Bone 13

2.3.4 Determinanten der Compliance bei Bluthochdruck 14

2.3.5 Zusammenhang zwischen Compliance und Blutdruckeinstellung 16

2.4 Hausärztliche Versorgung von Patienten mit Bluthochdruck 17

$\begin{array}{lr}\text { 3. Fragestellungen } & 19\end{array}$

4. Material und Methoden $\quad 20$

$\begin{array}{lll}4.1 & \text { Teilnehmende Praxen } & 20\end{array}$

4.2 Auswahl der Studienpatienten 21

4.3 Eingesetzte Fragebögen 25

4.3.1 Soziodemographische Datenerhebung 25

4.3.2 Fragen zum Blutdruck 25

4.3.3 Fragen zur Medikamenteneinnahme 25

4.3.4 Hill-Bone-Fragebogen zur Erhebung der Compliance 26

$\begin{array}{ll}4.4 & \text { Datenanalyse } \\ \end{array}$

$\begin{array}{ll}4.5 & \text { Datenschutz und Ethik }\end{array}$ 
5.1 Soziodemographische und anamnestische Charakteristika der Studienteilnehmer

5.2 Vergleich zwischen Studienteilnehmern mit optimaler vs. nicht optimaler Compliance

5.3 Unabhängigkeit der Variablen mit Einfluss auf die Compliance

6. Diskussion

6.1 Ausmaß der Compliance

6.2 Determinanten der Compliance

6.3 Compliance und Blutdruckeinstellung

6.3.1 Wirksamkeit der Therapie

6.3.2 Erhebung der Compliance

6.3.3 Erhebung der Blutdruckwerte

6.4 Repräsentativität der Studienteilnehmer

6.5 Bedeutung der Ergebnisse und Schlussfolgerung

7. Zusammenfassung

8. Literaturverzeichnis

9. Anhang

9.1 Ausschlusskriterien

9.2 Anschreiben an die Patienten vom Hausarzt

9.3 Antwortbogen Studienteilnahme

9.4 Aufklärung und Einverständniserklärung 


\section{Verzeichnis der Abbildungen}

Abbildung 1: Verlauf der Patientenrekrutierung

\section{Verzeichnis der Tabellen}

Tabelle 1: Soziodemographische und anamnestische Charakteristika der Studienteilnehmer

Tabelle 2: Vergleich zwischen Studienteilnehmern mit optimaler vs. nicht optimaler Compliance

Tabelle 3: Logistische Regression der vier Kovariaten mit signifikantem Einfluss auf die Compliance 


\section{Einleitung}

Die vorliegende Dissertation beschäftigt sich mit dem Thema der Compliance. Während des Medizinstudiums wird den Studenten bereits im vorklinischen Studienabschnitt die Theorie der Compliance vermittelt. Sie beschreibt einen Aspekt der Arzt-Patienten-Beziehung. Wörtlich übersetzt bedeutet der Begriff in etwa „Willfährigkeit“, „Unterwürfigkeit“ und „Einwilligung“. In den Kursen der psychosozialen Medizin wird gelehrt, dass diese Begriffe eine asymmetrische Arzt-Patienten-Beziehung charakterisieren, welche aber nachweislich zu einer schlechten Mitarbeit des Patienten in der Therapie führt. In der in den meisten Fällen anzustrebenden modernen Arzt-Patienten-Beziehung, in der Behandlungsentscheidungen nach dem Prinzip des shared decision-making von Arzt und Patient gemeinsam getroffen werden sollten, bedeutet Compliance das Mitwirken und die Mitarbeit des Patienten in der Therapieentscheidung und der Therapieumsetzung.

Die Compliance-Thematik ist ein Gebiet, auf dem intensiv geforscht wurde und weiterhin geforscht wird. In der Online-Datenbank PubMed liefert die Suche nach dem Begriff Compliance mehr als 89.000 Treffer. Trotz dieses großen wissenschaftlichen Aufwands und der hieraus gewonnenen Erkenntnisse, die auch bereits Eingang in die universitäre Lehre gefunden haben, erscheint mir die Umsetzung der Erkenntnisse im ärztlichen Alltag nicht optimal. Dies ist mein subjektiver Eindruck, den ich im Verlauf meines Medizinstudiums in diversen Famulaturen und Praktika, sowie in den ersten Monaten meiner ärztlichen Tätigkeit in einer internistischen Klinik gewonnen habe. Compliance wird nach meinem Erachten in der ärztlichen Praxis - wobei meine Erfahrungen insbesondere aus dem Bereich der stationären Patientenversorgung stammen - häufig als allein patienteninhärente Eigenschaft gesehen. Das Berücksichtigen von Erkenntnissen aus der Compliance-Forschung in der Kommunikation mit den Patienten könnte häufig zu einer für beide Seiten zufrieden stellenderen Behandlung führen.

Mit der vorliegenden Arbeit wird die Liste der wissenschaftlichen Beiträge zur Complianceforschung wieder etwas länger. Ich hoffe, die Ergebnisse dieser Dissertation stellen einen weiteren Mosaikstein im wissenschaftlichen Bild der Compliance dar. Darüber hinaus hoffe ich - Bezug nehmend auf den vorausge- 
henden Absatz - dass hiermit ein Beitrag zur kritischen Auseinandersetzung mit unserer Auffassung des Compliancebegriffs und der Umsetzung im ärztlichen Alltag geleistet wird. 


\section{Stand der Forschung}

\subsection{Der Begriff der Compliance}

Compliance wird in den gebräuchlichen medizinischen Lexika als Bereitschaft eines Patienten zur Zusammenarbeit mit dem Arzt bzw. zur Mitarbeit bei diagnostischen oder therapeutischen Maßnahmen definiert (Roche Lexikon Medizin 2003, Psychrembel 2004). In der wissenschaftlichen Literatur wird häufig die Definition von Haynes zitiert: „Unter dem Begriff ,Compliance' versteht man den Grad, in dem das Verhalten einer Person in Bezug auf die Einnahme eines Medikamentes, das Befolgen einer Diät oder die Veränderung des Lebensstils mit dem ärztlichen oder gesundheitlichen Rat korrespondiert" (Haynes 1982, S.12).

Der Begriff ,Compliance' ist umstritten. Bereits in den 1980er Jahren waren sich führende Compliance-Forscher darüber bewusst, dass durch den Begriff das Bild eines sündigen bzw. hörigen Patienten erweckt würde. Dies liefe der Entwicklung des Verhältnisses zwischen Patient und Arzt in der westlichen Gesellschaft entgegen. Trotzdem hielten sie an dem Begriff "Compliance" als Terminus technicus fest. Er sei schon zu tief verwurzelt und die Autoren sahen sich nicht in der Lage, ihm eine akzeptable Alternative entgegenzustellen (Haynes 1982). Ein anderer, in der neueren englischsprachigen Literatur häufig verwendeter Begriff, ist ,Adherence'. Allerdings ist auch er nicht frei von Kritik. Ihm wird ebenfalls angelastet, eine Anschauung zu repräsentieren, die hinderlich sei, das self-management der Patienten zu stärken (Glasgow und Anderson 1999, Steiner und Earnest 2000). In den letzten Jahren wurde von einigen Autoren die Verwendung des Begriffs ,Concordance' propagiert. Concordance unterscheide sich fundamental von Begriffen wie ,Compliance' und ,Adherence', weil eine entscheidend andere Sichtweise auf die Arzt-Patienten-Beziehung damit verbunden sei. Concordance beschreibe den gesamten Kommunikationsprozess und nicht ein spezifisches Patientenverhalten, der Begriff beinhalte shared decision-making und nicht ein paternalistisches Modell der Arzt-PatientenBeziehung (Weiss und Britten 2003).

Trotz dieser Kritik ist ,Compliance' nach wie vor der gebräuchlichste Begriff in der Literatur (Vermeire et al. 2001). Die in der vorliegenden Arbeit hauptsächlich behandelten Fragestellungen implizieren eher die traditionelle und engere 
Bedeutung der Compliance, nämlich in welchem Ausmaß der Patient die verschriebenen Medikamente einnimmt. Der Prozess der Therapiefindung zwischen Patient und Arzt, der mit dem Modell der Concordance zweifelsfrei angemessener beschrieben werden kann, wird nicht näher untersucht. Aus diesem Grund wird im folgenden Text der Begriff ,Compliance' verwendet.

Compliance ist kein stabiles Persönlichkeitsmerkmal des Patienten, sondern ein komplexes, dynamisches und situationsabhängiges Phänomen, das sich innerhalb eines Behandlungsplanes oder über den Behandlungsverlauf unterschiedlich manifestieren kann. Ein Patient kann in einigen Aspekten vollkommen compliant sein, während er zugleich andere Therapiebestandteile bzw. -schritte ablehnt oder mit deren Umsetzung überfordert ist (Cochrane 1996). Die vorliegende Arbeit beschäftigt sich in erster Linie mit der MedikamentenCompliance. Andere Aspekte der Compliance wie Lebensstiländerungen, das Einhalten von Arztterminen oder Ernährungsumstellungen werden nur gestreift.

\subsection{Die Bedeutung der Compliance in der evidenzbasierten Medizin}

Bereits 1976 merkte David Sackett an, dass für die ärztliche Entscheidung, Strategien zur Änderung des Compliance-Verhaltens anzuwenden, bestimmte Voraussetzungen erfült sein müssen. Unter anderem solle der Nutzen einer Therapie gegenüber einem möglichen Schaden überwiegen. Wenn die klinische Wirksamkeit der Therapie nicht eindeutig belegt sei, werden Patienten, die ihr Compliance-Verhalten ändern, lediglich erhöhten Behandlungsrisiken ausgesetzt, statt Nutzen aus der Behandlung zu ziehen (Sackett 1976). DiMatteo und Haskard formulierten es so: „Promoting adherence is a noble goal only if the treatment is correct." (DiMatteo und Haskard 2006, S.298).

lain Chalmers hat in einem Artikel einige Beispiele aus der Geschichte der Medizin aufgeführt, in denen die ärztliche Behandlung vielen Patienten mehr geschadet als genutzt hat (Chalmers 2006). Als ein Beispiel wird der Aderlass angeführt, der von einflussreichen Ärzten noch am Anfang des 20. Jahrhunderts als unerlässlich bei der Therapie der Pneumonie propagiert wurde. Ein weiteres Beispiel ist ein Ratschlag des bekannten Kinderarztes Dr. Benjamin Spock, der in seinem 1945 veröffentlichten und bis heute mehr als 50 Millionen Mal verkauften Bestseller ,Baby and Child Care' dazu riet, Säuglinge auf dem Bauch schlafen zu lassen. Er begründete diesen Ratschlag mit der Gefahr des Erstickens im Falle 
des Erbrechens bei Babys, die auf dem Rücken schlafen. Heute wissen wir, dass dieser Ratschlag, obwohl er theoretisch gut nachvollziehbar war, zehntausende plötzlicher Kindbetttode nach sich zog. Besonders tragisch ist, dass es bereits 1970 durch eine systematische Literaturrecherche möglich gewesen wäre, die Gefahren des Schlafens auf dem Bauch zu erkennen (Gilbert et al. 2005). Chalmers führt noch weitere Beispiele aus der jüngeren Vergangenheit an, wie zum Beispiel die Hormonersatztherapie bei Frauen in den Wechseljahren, die aufgrund unzuverlässiger Evidenz und aggressivem Marketing noch vor wenigen Jahren weit verbreitet war und die Frauen vor Herzinfarkten und Schlaganfällen bewahren sollte. Durch bessere Studien wurde jedoch bewiesen, dass die Hormontherapie das Risiko für diese kardiovaskulären Ereignisse sogar erhöht und darüber hinaus weitere unerwünschte Folgen nach sich ziehen kann (McPherson 2004).

Diese Beispiele machen deutlich, wie entscheidend wichtig es für den Arzt ist, die nach aktuellem Wissenstand beste Therapie zu kennen. Doch woher wissen Ärzte, welches die beste Therapie ist? Martin Edwards hat die Geschichte der Suche nach der besten Evidenz kurz umrissen (Edwards 2004). Im 19. und frühen 20. Jahrhundert galt die Meinung von angesehenen Ärzten als beste Evidenz. Im Jahre 1948 wurde die erste randomisiert-kontrollierte Studie (RCT) veröffentlicht und in den folgenden Jahren wurde diese Methode als Goldstandard für die beste Evidenz angesehen. In den 1970er Jahren schließlich fiel Kritikern wie Archibald Cochrane auf, dass Ärzte die Ergebnisse aus RCTs nur selektiv, zufällig oder unvollständig für ihre Arbeit übernahmen. Es resultierte die Bewegung der evidenzbasierten Medizin (EBM), die eine Hierarchie der Evidenz etablierte, an deren Spitze systematic reviews bzw. Metaanalysen stehen (Ebell et al. 2004).

Die EBM soll allerdings nicht als stures Befolgen von ,Kochrezepten' für die Therapie verstanden werden. David Sackett, einer der Pioniere auf diesem Gebiet, definiert EBM folgendermaßen: „Evidenzbasierte Medizin ist der gewissenhafte, eindeutige und vernünftige Gebrauch derzeitiger bester Evidenz für die Entscheidungsfindung in der Behandlung individueller Patienten. Die Ausführung von evidenzbasierter Medizin bedeutet die Integration von individueller klinischer Erfahrung und der besten verfügbaren externen Evidenz aus systematischer Forschung." (Sackett et al. 1996, S.71). 


\subsection{Die Rolle der Compliance bei der Bluthochdruckbehandlung}

Aus dem letzten Kapitel wird deutlich, dass höhere Compliance der Patienten mit der medikamentösen Therapie umso erstrebenswerter ist, je schwerwiegender ihr gesundheitliches Problem ist und je mehr die Therapie den Patienten nützt. Ein bedeutender Risikofaktor, auf den diese Bedingungen zutreffen, ist der Bluthochdruck. Seine gesundheitlichen Folgen und Therapiemöglichkeiten wurden intensiv untersucht, so dass mittlerweile verlässliche Erkenntnisse vorliegen.

\subsubsection{Prävalenz und Pharmakotherapie des Bluthochdrucks}

Die arterielle Hypertonie ist aufgrund ihrer Folgeerkrankungen, wie der koronaren Herzkrankheit und dem Schlaganfall, weltweit ein großes Gesundheitsproblem. Sie ist die Erkrankung mit der höchsten Mortalität und drittgrößter Risikofaktor für disability-adjusted life years (Ezzati et al. 2003). Insgesamt 26\% aller Erwachsenen weltweit, d.h. 972 Millionen Menschen, waren im Jahr 2000 erkrankt. Für das Jahr 2025 wird mit einer Steigerung der Prävalenz auf 29\% gerechnet, mit dann 1,56 Milliarden Betroffenen (Kearney et al. 2005). In Deutschland betrug die Prävalenz unter den 18- bis 79-Jährigen im Jahr 1998 bei Männern 30\% und bei Frauen 27\% (Thamm 1999). Im internationalen Vergleich von sechs europäischen Ländern, den USA und Kanada war in Deutschland in allen Altersgruppen sowohl die Prävalenz der Hypertonie als auch der mittlere arterielle Blutdruck am höchsten (Wolf-Maier et al. 2003).

In diversen Studien sind die Vorteile einer blutdrucksenkenden Therapie bewiesen worden. In den Richtlinien der European Society of Hypertension (ESH) und der European Society of Cardiology (ESC) sind zahlreiche randomisiertkontrollierte Studien und Meta-Analysen zu dieser Thematik zitiert und die Ergebnisse zusammengefasst worden (Mancia et al. 2007). Demnach sinkt durch antihypertensive Behandlung die Morbidität und Mortalität kardiovaskulärer Erkrankungen. Das Risiko für einen Schlaganfall wird um 30-40\% reduziert, das Risiko für Koronarerkrankungen um 20\%. In den Studien zeigte sich, dass es bei gleicher Blutdrucksenkung für Morbidität und Mortalität nahezu unerheblich ist, welche Medikamentenklasse therapeutisch eingesetzt wird. Es wird empfohlen, den Blutdruck bei arterieller Hypertonie auf mindestens 140/90mmHg zu senken und wenn es von den Patienten toleriert wird, auch darüber hinaus. Bei Patienten 
mit Diabetes mellitus oder anderen Risikofaktoren, wie bereits erfolgtem Schlaganfall, bereits erfolgtem Myokardinfarkt oder Proteinurie, betragen die Zielwerte 130/80mmHg oder niedriger.

\subsubsection{Erhebung der Compliance von Patienten mit Bluthochdruck}

Es gibt verschiedene Möglichkeiten, die Verfahren zur Messung der Compliance zu klassifizieren. Weit verbreitet und einfach ist die Einteilung in „direkte“ und „indirekte“ Messverfahren in Anlehnung an Gordis (1976). Zu den direkten Messverfahren werden z.B. die direkte Beobachtung der Medikamenteneinnahme oder die Bestimmung von Medikamenten bzw. Metaboliten in Blut, Urin, Stuhl oder Speichel gezählt. Beispiele für indirekte Verfahren sind Arzneimittelschwundmessungen (Rudd et al. 1988), elektronische Monitorsysteme und Patientenfragebögen oder -interviews (Osterberg und Blaschke 2005). Jedes dieser Verfahren hat Vor- und Nachteile und nicht jede Methode kann in jeder Situation sinnvoll eingesetzt werden. Die Frage nach dem besten Weg der Compliancemessung ist weiterhin offen. Schon 1979 wies Rudd in einem Artikel mit dem Titel "In search of the gold standard for compliance measurement" darauf hin, dass es nicht die eine optimale Methode der Compliancebestimmung für jede Fragestellung gebe, es aber an der Zeit sei herauszufinden, welche Methode unter welchen Bedingungen am besten geeignet sei (Rudd 1979).

Im Folgenden werden verschiedene für die Erhebung der Compliance bei der Behandlung des Bluthochdrucks häufig angewandte Verfahren vorgestellt. Besonderes Augenmerk liegt dabei auf den Verfahren zur Erhebung von Patientenangaben.

a) Direkter Nachweis der Prüfsubstanz oder eines Metaboliten

Die Validität hängt ganz wesentlich von der Sensitivität und Spezifität der eingesetzten klinisch-chemischen Methoden und der Pharmakokinetik des zu prüfenden Arzneimittels ab. Viele Medikamente zeichnen sich durch große intraund interindividuelle Variation des Plasmaspiegels bei gleicher Dosis aus (Hasford et al 1998). Erschwerend kommt bei der Bluthochdrucktherapie die große Anzahl der verschiedenen eingesetzten Medikamente hinzu. Für jeden einzelnen Wirkstoff muss ein Nachweisverfahren etabliert werden, wodurch diese 
Methode der Compliancemessung sehr aufwändig und teuer ist. Gelingt allerdings der Nachweis mit einer validierten Methode, so kann man sicher sein, dass der Patient die Prüftherapie auch angewandt hat. Vergleichbare harte, richtig-positive Daten über die erfolgte Arzneimittelanwendung liefert kein anderes Verfahren. Eine Beurteilung, ob die verordnete Dosierung zu den vorgegebenen Zeitpunkten angemessen und regelmäßig angewendet wurde, ist mittels dieser Methode aber in der Regel nicht möglich.

b) Arzneimittelschwundmessung

Vorteile dieses Verfahrens sind Einfachheit, Schnelligkeit, Reliabilität der Messung und Kostengünstigkeit. Nachteile bestehen darin, dass der Patient Tabletten vertauschen kann oder vor Arztbesuchen Tabletten wegwirft, um den Eindruck einer guten Compliance zu erwecken. Aus diesen Gründen werden pill counts von verschiedenen Autoren nicht als gute Methode zur Compliancebestimmung angesehen (Pullar et al. 1989, Rudd et al. 1988, Cramer et al. 1989). Darüber hinaus bietet diese Methode keine Information darüber, zu welchen Zeitpunkten die Medikamente eingenommen werden oder ob sie etwa über mehrere Tage hintereinander nicht eingenommen werden (Osterberg und Blaschke 2005).

c) Messung eines erwarteten biologischen Effekts

In der Therapie des Bluthochdrucks wäre die Senkung des Blutdrucks unter der Therapie mit einem Antihypertensivum die nahe liegende Messgröße. Das Eintreten einer erwarteten Wirkung ist jedoch nur ein sehr restriktiv zu nutzender Indikator für Compliance, da er unter anderem eine fixe Compliance-WirkungsBeziehung voraussetzt, eine Bedingung, die fast nie erfüllt ist. Zudem sind biologische Effekte von vielen weiteren Faktoren als der Medikamentencompliance abhängig, wie dem natürlichen Verlauf der Erkrankung, psychosozialen Faktoren oder Begleiterkrankungen (Osterberg und Blaschke 2005).

d) Elektronische Monitorsysteme (MEMS)

Seit 1987 sind spezielle Behälter mit Mikrochips im Deckel kommerziell erhältlich, die das Einnahmeverhalten von Patienten unter oraler Medikation elektronisch aufzeichnen. Mit einem PC können die Daten aus dem Speicherchip ausgelesen und einer statistischen Auswertung zugänglich gemacht werden (Cramer et al. 1989). Durch elektronische Monitorsysteme kann das Einnahmeverhalten zeitlich 
genauer als bei anderen Verfahren kontrolliert werden, wobei es als indirektes Verfahren aber nicht dokumentiert, ob der Patient das richtige Medikament und die richtige Dosis einnimmt. Patienten könnten einen Behälter öffnen und das Medikament nicht einnehmen, falsche Mengen an Tabletten entnehmen oder die Daten verzerren, indem sie Medikamente in einem anderen Behälter aufbewahren oder mehrere Dosen auf einmal entnehmen (Osterberg und Blaschke 2005). In der Literatur werden elektronische Monitorsysteme trotzdem häufig als genaueste Methode der Medikamentencompliance-Erhebung angesehen (Osterberg und Blaschke 2005, Paterson et al. 2002).

e) Patientenangaben

Hierzu werden mündliche Patientenbefragungen, Fragebögen und Patiententagebücher gezählt. Diese Verfahren zur Complianceerhebung sind verhältnismäßig kostengünstig und mit geringem apparativen Aufwand durchführbar. Ein viel diskutiertes Problem ist allerdings die Möglichkeit, dass Patienten aus unterschiedlichen Gründen falsche Angaben machen. Immerhin spricht wenig dafür, dass zuverlässige Patienten sich selbst als „non-compliant“ ausgeben oder dass diejenigen, die Non-Compliance zugeben, die Unwahrheit sagen. Falsch-negative Beurteilungen dürften hiermit bei diesem Verfahren die Ausnahme bilden. Kritischer anzusehen ist die Güte der positiven Antworten. Hier kann es durch Antworten entsprechend der sozialen Erwünschtheit zu Verzerrungen kommen (Osterberg und Blaschke 2005). Hasford et al. (1998) gehen nach einem Vergleich mit anderen Messverfahren davon aus, dass Interviews die tatsächliche Compliance erheblich überschätzen. Dennoch vertreten sie die Ansicht, dass die wenig befriedigenden Ergebnisse dieses Ansatzes nicht zuletzt im Mangel an geeigneten Interviewstilen und schriftlichen Erhebungsinstrumenten begründet liegen. Hasford et al. sprechen sich für die weitere Entwicklung von Methoden auf diesem Gebiet aus und führen als gelungenes Beispiel ein von Morisky et al. entwickeltes Interview zur Compliance-Erhebung bei Hypertonie-Patienten an, da dessen Ergebnisse deutlich mit der Kontrolle des Blutdruckes assoziiert waren (Morisky et al. 1986). Patientenangaben zur Complianceerhebung werden auch von Osterberg und Blaschke aufgrund ihrer Einfachheit und Effektivität eine Berechtigung zugesprochen. Bei dieser Aussage beziehen sie sich auf Arbeiten von Walsh et al. und Haynes et al. (Haynes et al. 1980, Walsh et al. 2002). 
Garber et al. haben untersucht, ob die Ergebnisse von Methoden, die auf Selbstauskünften der Patienten beruhen, mit den Ergebnissen anderer Methoden übereinstimmen (Garber et al. 2004). Diesbezüglich scheinen Fragebogen- und Tagebuchmethoden besser als Interviewmethoden zu sein.

\subsubsection{Erhebung der Compliance nach Hill-Bone}

Neben weiteren Fragebögen zur Compliance bei Bluthochdruck (Cook et al. 2005, Morisky et al. 1986, Wetzels et al. 2006) ist die ,Hill-Bone Compliance to High Blood Pressure Therapy Scale' ein häufiger angewandtes Verfahren (Kim et al. 2000). Da diese Methode in der vorliegenden Studie eingesetzt wurde, folgt eine ausführlichere Darstellung des Instruments und dessen Evaluation.

Bei der Entwicklung und Auswahl der Items griffen die Autoren vor allem auf die Erfahrung eines Expertenkomitees, bestehend aus drei Ärzten und fünf Krankenschwestern, sowie auf vorhandene Literatur und bisherige eigene Erfahrungen in der Compliance-Forschung zurück.

Die Zuverlässigkeit des Fragebogens wurde von Kim et al. mittels zweier verschiedener Gruppen von Patienten ermittelt. Beide Gruppen setzten sich aus Patienten zusammen, die wegen ihres erhöhten Blutdrucks in ambulanter medizinischer Behandlung waren. In beiden Gruppen waren die Teilnehmer afroamerikanischer Abstammung und lebten im Stadtgebiet von Baltimore. Ihr Blutdruck betrug bei zwei separaten Messungen jeweils mindestens 140/90mmHg. In der ersten Gruppe waren alle Teilnehmer männlich und zwischen 18 und 55 Jahre alt. Die Gruppengröße betrug 309 Teilnehmer. Die zweite Gruppe setzte sich aus 718 Frauen und Männern zusammen, hier gab es keine Altersbeschränkung. Alle Teilnehmer erhielten den Fragebogen zweimal, die erste Gruppe im Abstand von einem Jahr, die zweite Gruppe im Abstand von drei Jahren. Zu diesen Zeitpunkten wurde jeweils auch der Blutdruck gemessen.

Im Anschluss wurden die Fragebögen ausgewertet und Reliabilität sowie Vorhersage-Validität für den Blutdruck zum Zeitpunkt der ersten Beantwortung des Fragebogens und zum Zeitpunkt des Follow-up ermittelt. Aus den Ergebnissen schlossen die Autoren auf die Reliabilität und Validität ihres Instruments für afro-amerikanische, erwachsene Studienteilnehmer. Sie räumten die Möglichkeit ein, für weitere Studien nur die Subskala zur Medikamentenadhärenz zu benutzen und begründeten dies mit der Abbildbarkeit in der Faktorenanalyse. 
Nachdem der Hill-Bone-Compliance-Fragebogen veröffentlicht wurde, führten Marie Krousel-Wood und Kollegen eine weitere Untersuchung zur Reliabilität des Instruments durch (Krousel-Wood et al. 2005). An dieser Querschnittsstudie nahmen 239 Patienten mit ambulant behandelter Hypertonie teil. Das Durchschnittsalter der Teilnehmer betrug 69 Jahre, die Hälfte war männlich, drei Viertel der Teilnehmer waren weißer Hautfarbe. Der Fragebogen wurde den Patienten bei einem Kontrolltermin in einer auf Hypertonie spezialisierten ambulanten Einrichtung ausgeteilt. Krousel-Wood et al. benutzten für die Bestimmung der Compliance die Medikamentenadhärenz-Subskala des Hill-Bone-Fragebogens. Die Ergebnisse der Subskala wurden dichotomisiert. Die Studienteilnehmer, die einen Score von neun Punkten erreichten, galten als ,optimal compliant', die Studienteilnehmer, deren Score größer als neun war, galten als ,nicht optimal compliant'. Nach der statistischen Auswertung schlossen die Autoren auf eine gute Reliabilität des Fragebogens, insbesondere der MedikamentenadhärenzSubskala. Die Validität wurde von Krousel-Wood et al. in dieser Studie nicht untersucht.

\subsubsection{Determinanten der Compliance bei Bluthochdruck}

In den letzten Jahrzehnten wurden viele Forschungsarbeiten veröffentlicht, in denen Determinanten der Compliance Gegenstand der Untersuchung waren. In ihrem Artikel "Variations in patients' adherence to medical recommendations: a quantitative review of 50 years of research." stellt Robin DiMatteo die Ergebnisse einer Metaanalyse von 569 Studien zur Compliance dar (DiMatteo 2004). In 164 der Studien wurden Korrelationen zwischen der Compliance und soziodemographischen Merkmalen wie Alter, Geschlecht, Bildung und sozioökonomischem Status untersucht. Insgesamt stellte DiMatteo einen nur geringen Einfluss von soziodemographischen Merkmalen auf die Compliance fest, der abhängig von der untersuchten Population, der Behandlung und den Messmethoden war. Für Methoden, denen Selbstauskünfte der Studienteilnehmer zur Compliance zugrunde lagen, sank die Compliance mit dem Alter, während sie bei anderen Messmethoden mit dem Alter anstieg. Das Geschlecht korrelierte nicht mit der Compliance. Eine Abhängigkeit der Compliance von der Bildung war vor allem bei chronischen Erkrankungen zu beobachten. Die Compliance korrelierte positiv mit der Bildung der Patienten. Für das Einkommen und den sozioökonomischen 
Status konnte ebenfalls eine positive Korrelation mit der Compliance nachgewiesen werden, vor allem wenn das Einkommen als Marker des sozioökonomischen Status herangezogen wurde.

Neben soziodemographischen Merkmalen wurden in weiteren Studien auch therapiespezifische Determinanten untersucht. Schroeder et al. fanden in einem systematic review von randomisiert kontrollierten Studien heraus, dass durch Verringerung der Anzahl täglicher Dosierungen eine bessere Compliance erreicht werden konnte (Schroeder et al. 2004). Kjellgren et al. stellten nach der Durchsicht von über 200 Studien zur Medikamenten-Compliance bei Bluthochdruck weitere Merkmale und ihre Bedeutung für die Compliance zusammen (Kjellgren et al. 1995). Die Ergebnisse waren in manchen Fällen widersprüchlich. So sollte einerseits eine mehr als fünf Jahre andauernde Arzneimitteleinnahme die Compliance positiv beeinflussen, während andererseits die Compliance mit anhaltender Behandlungsdauer abnehmen sollte. Das Auftreten von Nebenwirkungen sollte die Compliance vermindern, hatte aber in anderen Studien keinen Einfluss auf die Compliance gezeigt. Vor diesem Hintergrund fassen Vermeire et al. zusammen, dass mittlerweile fast 200 unterschiedliche Variablen aus den verschiedenen Bereichen untersucht wurden, aber keine von ihnen konsistent mit der Compliance zusammenhing oder gute Vorhersagen für die Compliance ermöglichte (Vermeire et al. 2001).

Zahlreiche Studien untersuchen das Ausmaß der Compliance bei der Behandlung des Bluthochdrucks. Ihre inhomogenen Ergebnisse lassen sich durch Unterschiede bei Messinstrumenten, Studienpopulationen und Definitionen von Compliance begründen. In der bereits erwähnten Metaanalyse zeigte DiMatteo, inwieweit die gemessenen Complianceraten von dem gewählten Messinstrument abhängen (DiMatteo 2004). Die mittleren Complianceraten waren mit $85 \%$ beim pill count am höchsten und mit $67 \%$ bei der Fremdbeurteilung am niedrigsten. Verfahren, die auf Patientenangaben beruhten, zeigten eine mittlere Compliance von $72 \%$. Insgesamt betrug der Durchschnitt der Compliance bei allen Messverfahren $75 \%$. Für die Behandlung des Bluthochdrucks hatte DiMatteo eine mittlere Compliance von $77 \%$ ermittelt. Mit der Medikamentencompliance-Subskala des Hill-Bone-Compliance-Fragebogens hatten Krousel-Wood et al. in ihrer Studie bei über 70\% der Teilnehmer perfekte Compliance festgestellt (Krousel-Wood et al. 2005). 


\subsubsection{Zusammenhang zwischen Compliance und Blutdruckeinstellung}

Es stellt sich die Frage, auf welche Weise und in welchem Ausmaß Medikamenten-Compliance und Blutdruckeinstellung miteinander verknüpft sind. Unter der rein theoretischen Annahme, dass 0\% Compliance mit der Therapie zu keiner Senkung des Blutdrucks führt und 100\% Compliance zur Senkung des Blutdrucks auf den therapeutisch optimalen Wert, gibt es unterschiedliche Modelle für die Werte zwischen 0\% und 100\% Compliance. Die Blutdrucksenkung könnte linear mit der Compliance zunehmen, exponentiell oder sigmoid verlaufen oder sich noch anders verhalten. Kravitz und Melnikow (2004) geben als Beispiel für das lineare Modell die Bluthochdrucktherapie an. Allerdings dürfte für die Bluthochdrucktherapie eine allgemeine Aussage schwierig sein, da diverse Medikamente mit unterschiedlichen Wirkmechanismen und unterschiedlicher Pharmakokinetik eingesetzt werden.

Bramley et al. (2006) untersuchten in einer Studie den Zusammenhang von Blutdruckkontrolle und Compliance mit antihypertensiver Monotherapie. In dieser Studie wurde anhand des Anteils eingelöster Rezepte über einen längeren Zeitraum die Compliance der 840 Studienteilnehmer bestimmt. Hiervon waren $75 \%$ sehr compliant, $20 \%$ mittelmäßig compliant und $5 \%$ wenig compliant. Von den Patienten mit hoher Compliance erreichten 43\% Blutdruckwerte von unter 140/90mmHg. In den anderen beiden Gruppen betrug dieser Anteil 34\% bzw. 33\%. Patienten mit hoher Compliance erreichten optimale Blutdruckwerte knapp 1,5-mal so häufig wie Patienten mit mittlerer und niedriger Compliance (Odds Ratio $=1,45$ in der logistischen Regression). Die relativ niedrige Rate gut eingestellter Patienten, sogar unter denjenigen mit hoher Compliance, erklärten die Autoren mit der ungenügenden Monotherapie.

Eine Studie von Burnier et al. (2001) zeigte, dass auch bei der Therapie mit mehreren Bluthochdruckmedikamenten die Compliance Auswirkungen auf den erreichten Blutdruck hat. In dieser Studie wurde bei Patienten mit therapierefraktärem Bluthochdruck die Compliance mit elektronischen Messsystemen bestimmt. Der Blutdruck der Studienteilnehmer sank allein durch die Compliancemessung signifikant, ohne dass die Therapie modifiziert wurde. Die Autoren schlossen aus ihren Ergebnissen, dass die experimentelle Kontrolle die 
Compliance erhöhte und diese Erhöhung zu einer Verbesserung der Blutdruckwerte führte.

Nicht nur eine generell niedrige Compliance bedingt einen suboptimal eingestellten Blutdruck. Auch die pathophysiologischen Konsequenzen einer inkonsistenten Einnahme müssen diskutiert werden (Cramer 2002). Das Auslassen einer Dosis eines kurzwirksamen Calciumkanalblockers oder BBlockers führte in einer Studie zu signifikant erhöhten Blutdruckwerten und erhöhter Herzfrequenz während der folgenden zwei Tage. Einige Patienten entwickelten in der Folge übermäßig erhöhte Blutdruckwerte (ReboundPhänomen) (Johnson und Whelton 1994). In einer weiteren Studie wurde ebenfalls ein Rebound-Phänomen des Blutdrucks beobachtet, nachdem ßBlocker wie Propanolol oder Metoprolol plötzlich abgesetzt wurden (Rangno und Langlois 1982).

\subsection{Hausärztliche Versorgung von Patienten mit Bluthochdruck}

Die hausärztliche Praxis ist der Ort, in dem die Hypertonie diagnostiziert und behandelt wird (Donner-Banzhoff 2001). Die Punktprävalenz für Bluthochdruck in 1912 hausärztlichen Praxen in Deutschland betrug in einer im September 2001 durchgeführten Studie 42\% (Steckelings et al. 2004). Die meisten Menschen mit Bluthochdruck spüren von ihrer Erkrankung nichts und nur 37\% von innen sind sich ihrer Erkrankung bewusst (Wolf-Maier et al. 2004). In Deutschland erhalten nur 26\% der Menschen mit Hypertonie eine medikamentöse Behandlung (WolfMaier et al. 2004). Aufgrund der bereits angesprochenen evidenten Vorteile der Therapie für die Betroffenen kann diese Behandlungsrate nicht zufrieden stellen. Die Rate der Menschen mit Hypertonie, deren Blutdruck unter der angestrebten Grenze von 140/90mmHg eingestellt ist, beträgt in Deutschland nur 8\% (WolfMaier et al. 2004). In hausärztlichen Praxen beträgt diese Rate immerhin 19\% (Steckelings et al. 2004). Trotzdem deutet eine solch niedrige Rate an kontrolliertem Bluthochdruck, insbesondere unter Patienten, die sich in ärztlicher Behandlung befinden, auf großen Handlungsbedarf hin. Steckelings et al. (2004) führen die alarmierenden Ergebnisse auf überholte Behandlungsziele der Ärzte zurück, die nicht nach den aktuellen Leitlinien agierten. Donner-Banzhoff (2001) sieht das Problem hingegen nicht einfach nur in einem zu langsamen und lückenhaften Transfer von Forschungsergebnissen in die Praxis, sondern in 
strukturellen Hindernissen, welche einer überlieferten Behandlungsstrategie Grenzen setzten. Unter anderem sieht er ein Problem darin, dass mindestens $30 \%$ der verschriebenen Medikamentendosen nicht genommen würden. Diese stille Weigerung der Patienten am Projekt „Blutdruckkontrolle“ mitzuwirken, führt Donner-Banzhoff auf diskordante Behandlungsziele von Patient und Arzt zurück. 


\section{Fragestellungen}

\section{Ausmaß der Compliance hausärztlicher Patienten mit Bluthochdruck}

Aus den bisherigen Ausführungen wird deutlich, dass die Versorgung von hausärztlich betreuten Bluthochdruckpatienten in Deutschland nicht optimal ist. Ein viel diskutierter Grund hierfür liegt in mangelnder Compliance der Patienten mit der ärztlich verordneten medikamentösen Therapie. Das Ausmaß der Compliance bei Bluthochdruckpatienten wurde in Studien mehrfach untersucht. Für Deutschland, und gerade im hausärztlichen Bereich, gibt es allerdings keine entsprechende Studie. Eine Fragestellung, die mit der vorliegenden Arbeit beantwortet werden soll, betrifft also das Ausmaß der Compliance unter hausärztlich behandelten Bluthochdruckpatienten.

\section{Determinanten der Compliance hausärztlicher Patienten mit Bluthochdruck}

Die Determinanten der Compliance sind sehr häufig und mit widersprüchlichen Ergebnissen untersucht worden - abhängig von der Stichprobe und dem angewandten Erhebungsinstrument. Über die Patientenmerkmale scheint keine allgemeingültige Aussage möglich zu sein. Deswegen ist es von Interesse, gerade für die behandelnden Hausärzte, ob es spezifisch in der Population deutscher Bluthochdruckpatienten in hausärztlicher Behandlung anamnestische und soziodemographische Charakteristika gibt, die ein größeres Risiko für mangelnde Compliance bedeuten.

Klinische Bedeutung der Compliance hausärztlicher Patienten mit Bluthochdruck Kim et al. (2000) haben bei der Entwicklung des Compliance-Fragebogens dessen Validität mit Blutdruckmessungen an den Studienteilnehmern belegt. Sie haben herausgefunden, dass Personen mit eingestelltem Bluthochdruck bessere Compliancewerte im Fragebogen erreichten, als Personen, deren Bluthochdruck nicht eingestellt war. Krousel-Wood et al. (2005) haben nur die Medikamentencompliance-Subskala zur Complianceerhebung verwendet, die gemessenen Compliancewerte allerdings nicht mit Blutdruckwerten verglichen. Es stellt sich die Frage, ob die Medikamentencompliance mit der Blutdruckeinstellung korreliert und wie groß die Differenz der Blutdruckwerte von Personen mit optimaler und nicht optimaler Compliance ist. 


\section{Material und Methoden}

Für die Studie sollten Patienten mit der Diagnose ,Arterielle Hypertonie' gewonnen werden. Die Teilnehmer wurden mit Hilfe elektronischer Patientendaten aus mehreren Allgemeinarztpraxen im Raum Göttingen ausgewählt. Die Befragung der Teilnehmer erfolgte mittels eines Fragebogens, der den Teilnehmern per Post zugestellt wurde.

\subsection{Teilnehmende Praxen}

\section{Schritt 1: Auswahl der Praxen}

Es wurden insgesamt 119 Allgemeinarztpraxen und Praxen hausärztlich tätiger Internisten im Umkreis von $30 \mathrm{~km}$ um Göttingen angeschrieben und über die geplante Studie informiert. Daraufhin erklärten sich 40 Praxen zur Teilnahme bereit, hiervon mussten 10 Praxen wegen EDV-Problemen ausgeschlossen werden. Von den 30 ausgewählten Praxen befanden sich $18 \mathrm{im}$ Göttinger Stadtgebiet und 12 im Göttinger Umland.

Schritt 2: Datenexport

Die Daten für die Untersuchung sollten über die BDT (BehandlungsDatenTransfer)-Schnittstelle der Praxisverwaltungssoftware gewonnen werden. Das BDTFormat ist ein Standardformat, das unabhängig von der verwendeten Praxissoftware ist und in seiner ursprünglichen Version als AbrechnungsDatenTransfer (ADT)-Format von der Kassenärztlichen Bundesvereinigung zur elektronischen Quartalsabrechnung entwickelt wurde. Der Export von BDT-Daten aus der Praxis-EDV zum Zwecke wissenschaftlicher Studien ist ein etabliertes Verfahren (Himmel et al. 2006, Hummers-Pradier et al. 2003). Der Datenexport wurde von Mai 2005 bis Dezember 2006 durch EDV-Mitarbeiter der Abteilung Allgemeinmedizin der Universität Göttingen durchgeführt. Durch eine Pseudonymisierung direkt beim Export wurden die Personalien der Patienten aus Datenschutzgründen gelöscht. Lediglich die praxisinterne Patientennummer, die von der PraxisEDV beim Anlegen eines neuen Patienten vergeben wird, wurde beibehalten und ermöglichte eine spätere Reanonymisierung von ausgewählten Patienten. Bei sechs der ausgewählten 30 Praxen gelang der Datenexport aufgrund technischer Probleme nicht, die Daten zweier Praxen waren nahezu identisch, da es sich vormals um eine Gemeinschaftspraxis handelte. Es wurden nur die Daten einer 
dieser beiden Praxen exportiert. Insgesamt konnten somit die Daten aus 23 Praxen für die Studie erhoben werden. Hiervon befanden sich $13 \mathrm{im} \mathrm{Stadtgebiet}$ Göttingens und zehn im Göttinger Umland. Die Daten der 23 Praxen wurden in eine relationale Datenbank auf dem gesicherten Server der Abteilung Allgemeinmedizin importiert.

\subsection{Auswahl der Studienpatienten}

Aus dem Patientenstamm der teilnehmenden Praxen wurden in drei Schritten diejenigen Patienten herausgefiltert, die das Einschlusskriterium erfüllten und bei denen keine Ausschlusskriterien vorlagen.

\section{Schritt 1: Standardisierte elektronische Abfrage}

Der erste Schritt bestand aus einer standardisierten elektronischen Abfrage. Die vorhandenen anonymisierten BDT-Datensätze enthielten die Diagnosen und Behandlungsmaßnahmen aller dokumentierten Patientenkontakte des behandelnden Allgemeinarztes aus den letzten Jahren. Als nächstes mussten die Patienten mit der Diagnose ,Arterielle Hypertonie' identifiziert werden. Zu diesem Zweck wurde eine elektronische SQL-Abfrage (Structured Query Language) der BDT-Daten durchgeführt. Es galten folgende Einschlusskriterien: Alter $\geq 18$ Jahre und $\leq 80$ Jahre; Freitextdiagnose ,Arterielle Hypertonie' sowie entsprechende Synonyma; ICD-10 Diagnose 110 ,essentielle Hypertonie' (Graubner 2008); aktiver Patient in den letzten 12 Monaten (mind. eine Konsultation in diesem Zeitraum). Als Ausschlusskriterium bei der SQL-Abfrage galt die Diagnose ,Asthma bronchiale', um Überschneidungen mit einer weiteren, zeitgleich durchgeführten Studie der Abteilung Allgemeinmedizin zu vermeiden.

Die elektronische Abfrage lieferte 6617 Patientendatensätze, die den Suchkriterien entsprachen.

\section{Schritt 2: Standardisierte manuelle Durchsicht der Patientendatensätze}

In einem zweiten Schritt wurden die Patientendatensätze standardisiert manuell durchgesehen. Zu diesem Zweck wurden die Datensätze der bisher identifizierten 6617 Patienten aus den 23 Praxen in eine Excel-Tabelle übertragen. Diese Tabelle enthielt neben der anonymisierten Patientennummer Geburtsdatum, Geschlecht, Diagnosen mit Datum der Diagnosestellung und ICD-10-Schlüssel, Medikation und Notizen des behandelnden Arztes über den Behandlungsverlauf. 
Diese Tabelle wurde nun Praxis für Praxis manuell durchgesehen, um $\mathrm{Pa}$ tienten mit weiteren Ausschlusskriterien und die nach dem ersten Auswahlschritt falsch positiven Teilnahmekandidaten auszuschließen. Für die manuelle Durchsicht galten die im Anhang aufgeführten Ausschlusskriterien (siehe Anhang 9.1, Seite 58 f.). Im Falle eines Ausschlusses wurde dieser mit Begründung in der Excel-Tabelle dokumentiert. Wenn aus den Daten nicht eindeutig ersichtlich war, ob ein Patient auszuschließen war oder nicht, wurde der Datensatz für den anschließenden Praxisbesuch markiert.

\section{Schritt 3: Praxisbesuch und Re-Identifikation der Patienten}

Nach der manuellen Durchsicht der Excel-Tabelle mit den Patientendaten wurde die jeweilige Praxis besucht. Anhand der praxisinternen Patientennummer konnte in der Praxis der Patient wieder identifiziert werden. Mit den Praxismitarbeiterinnen wurde jeder Patient aus der Tabelle hinsichtlich der Ausschlusskriterien besprochen. Besonderes Augenmerk wurde auf die markierten Patienten gelegt, bei denen der Studieneinschluss aus den BDT-Daten allein nicht beurteilbar war. Die Praxismitarbeiterinnen konnten nach der Re-Identifikation in vielen Fällen Hinweise geben, ob die fraglichen Ausschlusskriterien zutrafen oder nicht. So wurde im dritten Schritt der Auswahl der Studienpatienten die Liste der möglichen Studienteilnehmer nach ihren Hinweisen aktualisiert.

Insgesamt erfüllten aus den 23 Praxen 3443 Patienten die Kriterien zum Studieneinschluss und wurden somit als geeignete Patienten zur Studienteilnahme eingeladen.

Dafür wurden die möglichen Studienteilnehmer der aktualisierten Liste im Namen des Hausarztes aus der Praxis heraus angeschrieben. Dieser erste Brief enthielt neben dem Anschreiben, in dem der potentielle Studienteilnehmer über den Inhalt der Studie informiert wurde, einen Antwortbogen und einen frankierten Rückumschlag an die Abteilung Allgemeinmedizin (siehe Anhang 9.2 und 9.3, Seite 60 f.).

Von den insgesamt 3443 angeschriebenen möglichen Studienteilnehmern schickten 1273, also gut ein Drittel, den Antwortbogen ausgefüllt zurück, hiervon waren 970 (28\% der angeschriebenen Personen) zu einer Teilnahme an der Studie bereit. Keine Antwort hingegen gaben 2170 Personen, also knapp zwei Drittel aller angeschriebenen Personen. 
Die 970 teilnahmebereiten Patienten erhielten einen zweiten Brief, der im Gegensatz zum ersten Brief mit der Abteilung Allgemeinmedizin der GeorgAugust-Universität Göttingen als Absender versandt wurde. Dieser Brief enthielt neben dem Anschreiben einen Fragebogen (siehe 4.3 Erhebungsinstrumente), eine Patientenaufklärung zur Studie, eine Einverständniserklärung zur Teilnahme an der Studie in zweifacher Ausfertigung und einen frankierten Rückumschlag an die Abteilung Allgemeinmedizin (siehe Anhang 9.4 und 9.5, Seite 62 bis 72). Von den 970 angeschriebenen Personen sendeten 75 Personen den Fragebogen nicht zurück, d.h. es lagen die zurückgesandten Fragebögen von 895 Studienteilnehmern vor.

In die Auswertung gingen 829 Datensätze ein; die Fragebögen von 66 Studienteilnehmern konnten nicht ausgewertet werden, da in 48 Fällen die Einverständniserklärung zurückgezogen wurde und in 18 weiteren Fällen die Angaben zur Medikamenteneinnahme fehlten. Abbildung 1 (siehe Seite 24) zeigt den Verlauf der Patientenrekrutierung im Überblick. 


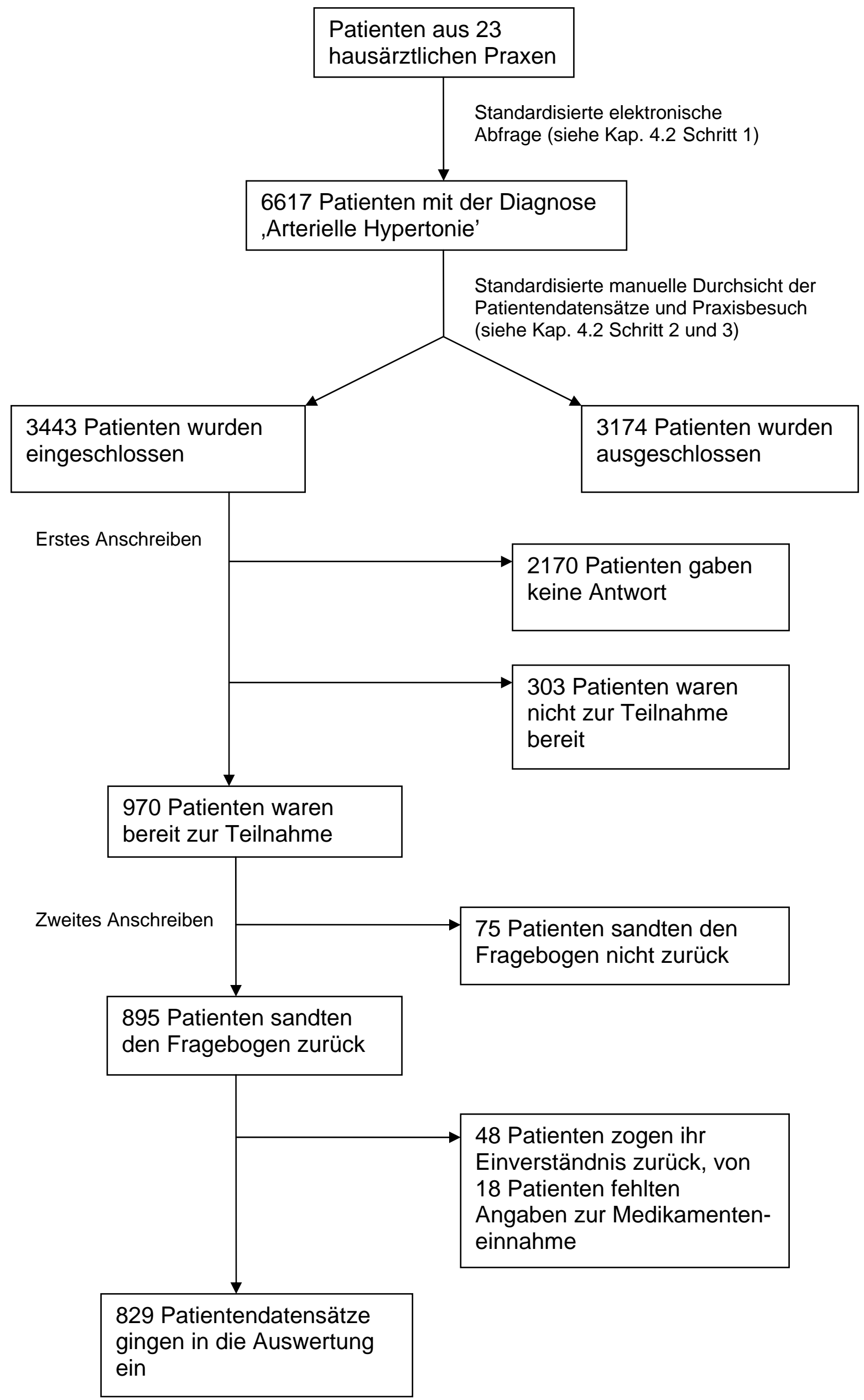




\subsection{Eingesetzte Fragebögen}

Der Umfang des Fragebogens betrug insgesamt acht Seiten (siehe Anhang 9.5, Seite 65 bis 72). Auf dem Deckblatt befand sich eine kurze Begrüßung der Studienteilnehmer, eine Aufklärung über die Freiwilligkeit der Beantwortung und den Datenschutz, sowie allgemeine Hinweise zum Ausfüllen des Fragebogens. Abschließend waren Kontaktdaten der Abteilung Allgemeinmedizin auf der ersten Seite aufgeführt. Die zweite und dritte Seite beschäftigten sich mit der Medikamenteneinnahme, auf der vierten Seite befanden sich Fragen zum Blutdruck. Die fünfte Seite bestand aus dem Fragebogen zur Compliance nach Hill-Bone (Kim et al. 2000). Die Seiten 6 bis 8 dienten der Erhebung der soziodemographischen Daten.

\subsubsection{Soziodemographische Datenerhebung}

Abgefragt wurden Informationen zum Geschlecht, zur Staatsangehörigkeit, zum Geburtsdatum, zu Partnerschaft und Familienstand, zur Haushaltsgröße, zur Schulbildung, zu Berufsabschlüssen, zur Erwerbstätigkeit und zum Haushaltseinkommen.

\subsubsection{Fragen zum Blutdruck}

Es wurden sechs anamnestische Fragen zum Blutdruck des Studienteilnehmers gestellt. In der ersten Frage sollte beantwortet werden, wie lange der zu hohe Blutdruck bekannt war. In der zweiten Frage sollte der Teilnehmer beantworten, seit wann er Medikamente gegen den hohen Blutdruck erhielt. Schließlich wurde nach der Person gefragt, die zuletzt den Blutdruck gemessen hatte. Die nächste Frage bezog sich darauf, wann der Blutdruck zuletzt gemessen wurde. In der fünften Frage wurde der Teilnehmer darum gebeten, den bei der letzten Blutdruckmessung ermittelten Wert einzutragen. In der sechsten und letzten Frage dieses Abschnitts wurde der Studienteilnehmer darum gebeten sich zu erinnern, wie hoch der Blutdruck vor Beginn der medikamentösen Therapie war.

\subsubsection{Fragen zur Medikamenteneinnahme}

In diesem Abschnitt wurden die Studienteilnehmer gebeten, ihre in den letzten sieben Tagen eingenommenen Medikamente einzutragen. Die Hinweise zum Ausfüllen des Medikamentenfragebogens beinhalteten die Abbildung einer 
Medikamentenschachtel mit Markierungen der wichtigen Informationen, die von der Schachtel abgelesen werden konnten, sowie eine beispielhafte Eintragung. Angegeben werden sollten: der Name des Medikaments, die Pharmazentralnummer (PZN), die Darreichungsform, die Dosis, der Grund für die Einnahme, die Packungsgröße (N1 bis N3), ob die Einnahme regelmäßig oder nach Bedarf erfolgt und ob sie vom Arzt verordnet wurde.

\subsubsection{Hill-Bone-Fragebogen zur Erhebung der Compliance}

Die fünfte Seite des Fragebogens diente der Erhebung der Compliance. In der Kopfzeile dieser Seite wurden die Studienteilnehmer darauf hingewiesen, dass es in den folgenden Fragen um die Einnahme von Blutdruckmedikamenten und um Gewohnheiten oder Probleme geht, die dabei eine Rolle spielen könnten. Die Fragen zur Compliance entsprachen einer deutschen Übersetzung der Hill-Bone Compliance to High Blood Pressure Therapy Scale (Kim et al. 2000). Da keine deutsche Übersetzung vorlag, wurde der Fragebogen in der Abteilung nach einem standardisierten Verfahren übersetzt, das sich in vereinfachter Form an dem IQOLA-Projekt orientierte (Bullinger et al. 1998). Der Fragebogen enthält 14 Items, die in drei Bereiche eingeteilt werden. Zwei Items betreffen das Einhalten von Arztterminen, drei Items nehmen Bezug auf den Kochsalzkonsum und die übrigen neun Items erfragen die Medikamentencompliance. Zu jedem Item gibt es vier vorgegebene Antwortmöglichkeiten, die unterschiedlich mit Punkten bewertet werden. Es sind insgesamt Werte von 14 bis 56 Punkten erreichbar, wobei ein niedriger Punktwert eine hohe Compliance anzeigt. Ein definitiver Cutoff Wert, der zwischen compliant und non-compliant differenziert, wurde von den Konstrukteuren des Instruments ursprünglich nicht festgelegt.

Zur Auswertung zogen wir allein die Medikamentencompliance-Subskala heran, wie es Kim et al. (2000) vorgeschlagen und Krousel-Wood et al. (2005) bereits durchgeführt haben. Hierbei unterschieden wir, analog zu der Arbeit von Krousel-Wood et al., zwischen optimaler Compliance und nicht optimaler Compliance. Als optimal compliant galten Studienteilnehmer, die alle neun Fragen zur Medikamentencomliace im Sinne höchster Compliance beantworteten. Wurde von Studienteilnehmern mindestens eine Frage nicht im Sinne höchster Compliance beantwortet, galten diese als nicht optimal compliant. 


\subsection{Datenanalyse}

Die Daten aus den von den Studienteilnehmern ausgefüllten und an die Abteilung Allgemeinmedizin zurückgesandten Fragebogen wurden in eine AccessDatenbank eingegeben. Die statistische Auswertung erfolgte mit dem Programm SPSS für Windows in der Version 14.0.

Zur statistischen Überprüfung von Hypothesen wurden Signifikanztests durchgeführt. Für die verhältnisskalierte Variable ,Alter' wurden Mittelwertunterschiede verschiedener Gruppen mittels t-Test auf Signifikanz überprüft. Für die Überprüfung nominalskalierter Variablen wurde eine Kreuztabelle angelegt. Mittels Chi²-Test wurde ermittelt, ob die beobachteten Häufigkeitsverteilungen signifikant von den erwarteten Häufigkeitsverteilungen abwichen.

Für die Überprüfung der unabhängigen Vorhersagewerte von Kovariaten für eine abhängige Variable wurde die logistische Regression als multivariates Verfahren eingesetzt. Kategoriale Variablen mit mehr als zwei Ausprägungen wurden dummycodiert. Als durchgehend mit Null codierte Referenzkategorie wurde hier die am häufigsten besetzte Kategorie gewählt. Bietet sich aus inhaltlichen Gründen keine der Gruppen als Referenzgruppe an, ist dies das empfohlene Vorgehen, da hierdurch die Varianz des Schätzers verringert werden kann (Kreienbrock und Schach 1995).

Das in allen statistischen Berechnungen festgelegte Signifikanzniveau lag bei $\alpha=5 \%$ (2-seitig getestet).

\subsection{Datenschutz und Ethik}

Patientinnen und Patienten, die für die Studie in Frage kamen, wurden über diese vollständig aufgeklärt und um ihre Einwilligung zur Teilnahme gebeten (informed consent). Die Anonymität der Patientendaten blieb erhalten; die Zusammenführung erfolgte lediglich über eine Kenn-Nummer. Im Vorfeld der Studie wurde das Vorgehen vom zuständigen Datenschutzbeauftragten überprüft.

Die Zustimmung der zuständigen Ethikkommission lag vor (Antragsnummer 23/4/05). 


\section{Ergebnisse}

\subsection{Soziodemographische und anamnestische Charakteristika der Studienteilnehmer}

Die Gruppe der Studienteilnehmer bestand aus 403 Frauen und 423 Männern; drei Studienteilnehmer hatten ihr Geschlecht auf dem Fragebogen nicht angegeben. Die Altersverteilung der Kohorte reichte von 20 bis 80 Jahre mit einem mittleren Alter von 61 Jahren (Standardabweichung 11 Jahre).

Für die Erhebung der Haushaltsgröße machten 826 Personen die erforderlichen Angaben. Gut drei Viertel der Studienteilnehmer lebte mit einem Partner zusammen, knapp ein Viertel lebte alleine.

Von den 829 Studienteilnehmern beantworteten 797 Personen die Frage zu abgeschlossenen Berufsausbildungen. Mit 492 Personen gab der größte Teil der Kohorte als höchsten Ausbildungsabschluss eine abgeschlossene betriebliche Berufsausbildung oder eine abgeschlossene Ausbildung an einer Fach-, Meister- oder Technikerschule an. Die zweitgrößte Gruppe, bestehend aus 130 Personen, hatte einen Hochschul- oder Fachhochschulabschluss. Beinahe ebenso groß war mit 121 Personen die Gruppe der Studienteilnehmer ohne beruflichen Abschluss. 53 Studienteilnehmer gaben eine andere abgeschlossene Berufsausbildung an, die nach ihrer Einschätzung mit den vorgegebenen Antwortmöglichkeiten nicht abgedeckt wurde. Eine Person befand sich zum Zeitpunkt der Erhebung in beruflicher Ausbildung (weiblich, 20 Jahre alt).

Für die Aussagen zum Status der Erwerbstätigkeit konnten die Angaben von 802 Personen herangezogen werden. Über die Hälfte der Studienteilnehmer waren Rentner, Pensionäre oder Vorruheständler, ein Viertel der Studienteilnehmer war in Vollzeit erwerbstätig, die übrigen Studienteilnehmer waren entweder in Teilzeit erwerbstätig oder nicht erwerbstätig. Der Gruppe der nicht Erwerbstätigen wurden Hausfrauen und -männer, arbeitssuchende Personen, Ein-Euro-Jobber, Frauen in Mutterschaftsurlaub und Auszubildende zugerechnet.

Neben soziodemographischen Angaben wurden auch anamnestische Informationen zum Bluthochdruck erhoben. Antworten auf die Frage, seit welcher Zeit die Bluthochdruckmedikation bestand, wurden in zwei Kategorien zusammengefasst. Die erste Kategorie fasste die Antworten bis fünf Jahre zusammen; 
in diese Gruppe fielen 327 Studienteilnehmer. Die übrigen 455 Studienteilnehmer waren laut Selbstauskunft bereits seit über fünf Jahren in medikamentöser Behandlung. Vierzig Teilnehmer gaben an, gar keine Medikamente gegen Bluthochruck zu erhalten.

Des Weiteren sollte beantwortet werden, wer den Blutdruck der Studienteilnehmer zuletzt gemessen hatte. 827 Personen machten zu dieser Frage Angaben. 461 hiervon hatten ihren Blutdruck zuletzt selbst gemessen, bei 365 Personen hatte jemand anderes den Blutdruck zuletzt gemessen und eine Person gab an, sich nicht erinnern zu können.

Auf die Frage, wann die letzte Blutdruckmessung erfolgte, antworteten 827 Studienteilnehmer. Bei über der Hälfte der Studienteilnehmer lag die letzte Blutdruckmessung weniger als eine Woche zurück, bei einem Viertel lag sie ein bis vier Wochen zurück, bei $12 \%$ ein bis drei Monate und bei sechs Prozent mehr als drei Monate. Ein Prozent der Befragten konnte sich nicht an den Zeitpunkt der letzten Blutdruckmessung erinnern.

Die Studienteilnehmer wurden im Fragebogen auch gebeten, die zuletzt bei ihnen gemessenen Blutdruckwerte anzugeben. 714 Personen gaben den systolischen und den diastolischen Wert an, zwei Personen nur den systolischen Wert und 86 Personen konnten sich nicht an die zuletzt gemessenen Werte erinnern. Der Mittelwert der systolischen Blutdruckwerte betrug $138 \mathrm{mmHg}$ mit einer Spannweite von 84 bis 205 mmHg und einer Standardabweichung von 15 $\mathrm{mmHg}$. Der diastolische Wert lag im Durchschnitt bei $82 \mathrm{mmHg}$ mit einer Spannweite von 51 bis $130 \mathrm{mmHg}$ und einer Standardabweichung von $10 \mathrm{mmHg}$. Bei 51\% der Befragten lag der systolische Blutdruck unter $140 \mathrm{mmHg}$ und bei 73\% der Befragten lag der diastolische Wert unter $90 \mathrm{mmHg}$. Insgesamt war der Blutdruck bei $45 \%$ der Studienteilnehmer mit Werten unter 140/90 mmHg eingestellt.

Tabelle 1 (Seite 30) gibt einen Überblick über die soziodemographischen und anamnestischen Charakteristika der Studienteilnehmer. 
Tabelle 1: Soziodemographische und anamnestische Charakteristika der Studienteilnehmer

\begin{tabular}{cc}
\hline Soziodemographische Charakteristika & \\
\hline Alter in Jahren, MW (SD) & $61(11)$ \\
Männliche Teilnehmer, \% & 51 \\
Haushaltsgröße, \% & \\
alleine lebend & 24 \\
mit Partner lebend & 76 \\
Berufsausbildung, \% & \\
Hoch-/Fachhochschule & 16 \\
Lehre/Fach- o. Meisterschule & 62 \\
andere Berufsausbildung & 7 \\
keine Berufsausbildung & 15 \\
Aktuelle Erwerbstätigkeit, \% & \\
in Vollzeit & 26 \\
in Teilzeit & 13 \\
Rentner/Vorruhestand & 53 \\
nicht erwerbstätig & 8 \\
\hline
\end{tabular}

Anamnestische Charakteristika ${ }^{1}$

Dauer der Blutdruckmedikation > 5 Jahre, \% 55

Letzte Messung Selbstmessung, \%

Zeitpunkt der letzten Messung, \%

innerhalb der letzten 7 Tage 56

vor 1-4 Wochen 26

vor 1-3 Monaten 12

vor mehr als 3 Monaten 6

keine Erinnerung 1

Zuletzt gemessener Blutdruck, \%

Systolisch $<140 \mathrm{mmHg} \quad 51$

Diastolisch $<90 \mathrm{mmHg} \quad 73$

$<140 / 90 \mathrm{mmHg} \quad 45$

MW Mittelwert; SD Standardabweichung

1 insgesamt n=829 Studienteilnehmer; Grundrate schwankt jedoch in Abhängigkeit zur vollständigen Beantwortung des jeweiligen Fragenkomplexes. 


\subsection{Vergleich zwischen Studienteilnehmern mit optimaler vs. nicht optimaler Compliance}

Für die Auswertung der Compliance wurden nur die Datensätze der Studienteilnehmer herangezogen, die nach eigenen Angaben Bluthochdruckmedikamente erhielten und die Fragen zur Compliance vollständig bearbeitet hatten. In der vorliegenden Untersuchung waren nach der unter Kapitel 4.3.4 (Seite 26) beschriebenen Definition 468 Studienteilnehmer optimal compliant (61\%) und 297 Studienteilnehmer nicht optimal compliant (39\%). 40 Studienteilnehmer erhielten nach eigenen Angaben keine Medikamente gegen Bluthochdruck und bei 24 Studienteilnehmern lag kein vollständiger Datensatz vor.

Studienteilnehmer mit optimaler Compliance und diejenigen mit nicht optimaler Compliance unterschieden sich bezüglich einiger soziodemographischer und anamnestischer Charakteristika: Studienteilnehmer mit nicht optimaler Compliance waren im Schnitt vier Jahre jünger und häufiger noch berufstätig. Sie lebten seltener mit einem Partner zusammen und nahmen eher erst seit weniger als fünf Jahren Medikamente gegen den Bluthochdruck ein. Keine Unterschiede ergaben sich hingegen bezüglich der Geschlechterverteilung und des Bildungsniveaus sowie bezüglich des Zeitpunkts oder der Art der letzten Blutdruckmessung (Fremd- vs. Selbstmessung). Auch bezüglich des systolischen bzw. diastolischen Blutdruckwerts laut Selbstauskunft ergaben sich keine Unterschiede zwischen Studienteilnehmern mit optimaler vs. nicht optimaler Compliance. Tabelle 2 (Seite 32) zeigt Mittelwerte bzw. Häufigkeiten der untersuchten Charakteristika in beiden Gruppen sowie die statistischen Kennwerte für die Prüfung signifikanter Unterschiede.

In einem Chi²-Test wurden schließlich die beiden dichotomen Variablen ,Blutdruck eingestellt unter 140/90 mm Hg' und ,Compliance' in Beziehung gesetzt. Die beobachteten Häufigkeiten wichen nur geringfügig von den erwarteten Häufigkeiten ab $\left(C h i^{2}=0,104 ; p=0,747\right)$. 
Tabelle 2: Vergleich zwischen Studienteilnehmern mit optimaler vs. nicht optimaler Compliance

Studienteilnehmer mit $^{1}$

$\begin{array}{ll}\text { optimaler } & \text { nicht opt. } \\ \text { Compliance } & \text { Compliance }\end{array}$

Soziodemographische Charakteristika

Alter in Jahren, $M W(S D)$

$63(10)$

$59(12)$

$-4,653 * * *$

Geschlecht, $n$

männlich

weiblich

237

161

230

134

1.061

Haushaltsgröße, $n$

alleine lebend

mit Partner lebend

$99 \quad 81$

$368 \quad 214$

3,925 *

Berufsausbildung, $n$

Hoch-/Fachhochschule

Lehre/Fach- o. Meisterschule

66

286

57

andere Berufsausbildung

33

169

keine Berufsausbildung

64

17

5,758

Aktuelle Erwerbstätigkeit, $n$

in Vollzeit

in Teilzeit

Rentner/Vorruhestand

nicht erwerbstätig
91

54

270

40
45

100

46

119

22
28,318 ***

Anamnestische Charakteristika

Dauer der Blutdruckmedikation, $n$

höchstens 5 Jahre

mindestens 5 Jahre

172

148

296

147

$13,377 * \star \star$

Letzte Blutdruckmessung, $n$

Selbstmessung

Fremdmessung

281

186

161

136

2,647

Zeitpunkt der letzten Messung, $n$

innerhalb der letzten 7 Tage

vor 1-4 Wochen

271

121

164

51

78

vor 1-3 Monaten

vor mehr als 3 Monaten

keine Erinnerung

22

1

23

1

3.238

Zuletzt gemessener Blutdruck, MW (SD)

Systolisch $140 \mathrm{mmHg}$

Diastolisch $90 \mathrm{mmHg}$
$138(15)$

$82(10)$
138 (14)

83 (10)
0.239

$-1.666$

MW Mittelwert; SD Standardabweichung

${ }^{1}$ insgesamt $n=468$ Studienteilnehmer mit optimaler und $n=297$ mit nicht optimaler Compliance; Grundrate schwankt jedoch in Abhängigkeit zur vollständigen Beantwortung des jeweiligen Fragenkomplexes.

${ }^{2}$ T-Test oder Chi²-Test mit * $p<0,05 ;{ }^{* \star} p<0,01 ;{ }^{* \star *} p<0,001$ 


\subsection{Unabhängigkeit der Variablen mit Einfluss auf die Compliance}

In einem multivariaten Verfahren wurde überprüft, ob die vier Variablen, die einzeln einen signifikanten Unterschied bei der abhängigen Variable Compliance boten, unabhängig voneinander einen signifikanten Vorhersagewert für die Variable Compliance leisteten. Zu diesem Zweck wurde das Verfahren der logistischen Regression eingesetzt. Die zu überprüfenden Kovariaten waren das Alter, die Haushaltsgröße (alleine lebend oder mit Partner lebend), der Status der Erwerbstätigkeit (in Vollzeit erwerbstätig, in Teilzeit erwerbstätig, Rentner oder nicht erwerbstätig) und die Dauer der Bluthochdruckmedikation (bis zu fünf Jahre oder über fünf Jahre). Die Kovariaten wurden im Einschlussverfahren geprüft.

Die Grundlage für die logistische Regression bildeten 738 Fälle. Für die Variablen ,Haushaltsgröße', ,Status der Erwerbstätigkeit' und ,Dauer der Bluthochdruckmedikation' konnte ein von den anderen Variablen unabhängiger signifikanter Vorhersagewert für die abhängige Variable ,Compliance' nachgewiesen werden. Die Variable ,Alter' bot keinen signifikant unabhängigen Vorhersagewert (siehe Tabelle 3, Seite 34). Die Ergebnisse bezüglich der Haushaltsgröße zeigen, dass Patienten, die mit einem Partner zusammenlebten, mit größerer Wahrscheinlichkeit compliant waren als Patienten, die alleine in einem Haushalt lebten. Analog hierzu waren Rentner eher compliant als in Vollzeit erwerbstätige Personen und Patienten mit mehr als fünfjähriger Medikamenteneinnahme eher compliant als Patienten mit kürzerer Medikamentenhistorie. 
Tabelle 3: Logistische Regression der vier Kovariaten mit signifikantem Einfluss auf die Compliance

\begin{tabular}{|c|c|c|c|}
\hline Variable & Differenz für OR & OR & $95 \% \mathrm{Cl}$ \\
\hline Alter & $1 \mathrm{Jahr}$ & 1,017 & $0,995-1,039$ \\
\hline Haushaltsgröße & $\begin{array}{l}\text { allein lebend vs. mit Partner } \\
\text { lebend }\end{array}$ & 0,680 & $0,475-0,975$ \\
\hline \multirow[t]{5}{*}{ Status der Erwerbstätigkeit } & Vollzeit-erwerbstätig vs. & 0,548 & $0,325-0,925$ \\
\hline & Rentner & & \\
\hline & Teilzeit-erwerbstätig vs. & 0,680 & $0,391-1,181$ \\
\hline & Rentner & & \\
\hline & nicht erwerbstätig vs. Rentner & 1,165 & $0,591-2,294$ \\
\hline $\begin{array}{l}\text { Dauer der Bluthochdruck- } \\
\text { medikation }\end{array}$ & über 5 Jahre vs. bis 5 Jahre & 1,597 & $1,170-2,178$ \\
\hline
\end{tabular}

OR Odds-Ratio; Cl Konfidenzintervall 


\section{Diskussion}

Die wichtigsten Resultate der vorliegenden Arbeit lassen sich folgendermaßen zusammenfassen: $61 \%$ der Studienteilnehmer waren optimal compliant. Die Studienteilnehmer, die nicht im Sinne optimaler Compliance antworteten, waren im Schnitt vier Jahre jünger und häufiger noch berufstätig als Studienteilnehmer mit optimaler Compliance. Sie lebten seltener mit einem Partner zusammen und nahmen eher erst seit weniger als fünf Jahren Medikamente gegen den Bluthochdruck ein. Besonders bemerkenswert war, dass sich die Blutdruckwerte von Personen mit optimaler Compliance nicht von den Blutdruckwerten von Personen mit nicht optimaler Compliance unterschieden.

Die Datengrundlage bildeten Selbstauskünfte der Studienteilnehmer aus Göttingen und dem Umland, die mit Hilfe eines Fragebogens erhoben wurden. Für die Bestimmung der Compliance kam der Compliance-Fragebogen nach HillBone zum Einsatz, dessen Medikamenten-Compliance-Subskala ausgewertet wurde.

\subsection{Ausmaß der Compliance}

In der vorliegenden Studie waren 61\% der Studienteilnehmer optimal compliant. Vergleichszahlen für deutsche hausärztliche Patienten mit Bluthochdruck liegen nicht vor, es muß also auf internationale Studien zurückgegriffen werden. Zunächst bietet sich die Arbeit von Krousel-Wood et al. (2005) an, in der zur Complianceerhebung das gleiche Instrument eingesetzt wurde. In dieser Studie waren über $70 \%$ der Befragten optimal compliant. Zu berücksichtigen sind bei dem Vergleich beider Ergebnisse allerdings Unterschiede in der Durchführung und der Patientenstichprobe. Krousel-Wood et al. händigten den Fragebogen an Patienten aus, die die Sprechstunde einer auf Hypertonie spezialisierten internistischen Abteilung einer großen Gemeinschaftspraxis aufsuchten. Aus dem Artikel wird nicht deutlich, ob alle Patienten von einem einzigen Arzt behandelt wurden oder ob mehrere Ärzte in der Abteilung arbeiteten. Die Studienteilnehmer beantworteten den Fragebogen in der Arztpraxis und nicht, wie in der vorliegenden Studie, bei sich zu Hause. Bei der Patientenstichprobe von Krousel-Wood et al. handelte es sich um Bluthochdruckpatienten im amerikanischen Gesundheitssystem mit einem mittleren Alter von 69 Jahren gegenüber einem Durchschnitts- 
alter von 61 Jahren in der vorliegenden Studie mit deutschen Bluthochdruckpatienten. Das Geschlechterverhältnis war in beiden Studien ausgeglichen. Für die höhere Compliancerate in der amerikanischen Studie gibt es mehrere plausible Begründungen. Die Teilnehmer in der Studie von Krousel-Wood et al. waren im Schnitt acht Jahre älter als in der vorliegenden Studie. In beiden Studien konnte nachgewiesen werden, dass ältere Personen den Fragebogen eher im Sinne optimaler Compliance ausfüllten als jüngere Personen. Krousel-Wood et al. merkten in der Diskussion ihrer Ergebnisse an, dass ihre Patientenstichprobe in einer auf Hypertonie spezialisierten Einrichtung rekrutiert wurde und deshalb diese Patienten möglicherweise schwerer erkrankt seien, anders behandelt würden und besser motiviert seien, der Therapie zu folgen, als Patienten in hausärztlicher Behandlung. Des Weiteren muss auch berücksichtigt werden, dass die Studien in zwei unterschiedlichen Gesundheitssystemen durchgeführt wurden. Es konnte gezeigt werden, dass amerikanische Patienten im Vergleich zu deutschen Patienten sich ihres erhöhten Blutdrucks viel häufiger bewusst sind und dass der Anteil an Patienten mit kontrolliertem Blutdruck in den USA deutlich höher liegt als in Deutschland (Wolf-Maier et al. 2004). Dies deutet auf eine möglicherweise höhere Compliance unter amerikanischen Bluthochdruckpatienten im Vergleich zu deutschen hin.

Im Vergleich zu einer Metaanalyse von 129 Studien zur Compliance bei kardiovaskulären Erkrankungen lag die Rate optimaler Compliance der vorliegenden Studie ebenfalls unter dem dort ermittelten Durchschnittswert von 77\% (DiMatteo 2004). Neben den bereits im vorherigen Absatz angesprochenen möglichen Gründen für abweichende Werte kommen hier weitere Faktoren hinzu. In der Metaanalyse wurden Studien mit verschiedenen Messmethoden für die Complianceerhebung zusammengetragen. Bei einigen wird die Compliance höher eingeschätzt als bei Fragebogenerhebungen, mit denen durchschnittlich eine Compliance von $72 \%$ gemessen wird (DiMatteo 2004). Außerdem muss berücksichtigt werden, dass in der vorliegenden Studie die Rate optimaler Compliance gegenüber nicht optimaler Compliance erhoben wurde. Dabei galt ein sehr strenges Kriterium für optimale Compliance, nämlich dass nach Selbstauskunft der Studienteilnehmer gar keine Medikamenteneinnahme versäumt wurde. Die in anderen Studien verwandten Definitionen von Compliance weichen in den meisten Fällen von dieser Definition ab - sie sind nicht so 
streng. Die direkte Vergleichbarkeit der Compliancerate mit Studien, in denen per pill count oder mit elektronischen Messungen das Verhältnis von eingenommener zu verschriebener Medikation ermittelt wurde, ist ebenfalls nicht gegeben.

\subsection{Determinanten der Compliance}

In der vorliegenden Studie wurden einige Patientencharakteristika und ein Behandlungsmerkmal als Prädiktoren für nicht optimale Compliance identifiziert. Studienteilnehmer, die jünger und noch berufstätig waren, die alleine lebten und die seit weniger als fünf Jahren Bluthochdruckmedikamente einnahmen, waren eher nicht optimal compliant (siehe Tabelle 2, Seite 32).

In der Studie von Krousel-Wood et al. (2005) war das einzig signifikante Ergebnis in diesem Zusammenhang die höhere Wahrscheinlichkeit von weißen gegenüber nicht weißen Personen, optimal compliant zu sein. Studienteilnehmer über 65 Jahre, Männer, Personen mit High-School Ausbildung und Verheiratete schienen eher optimal compliant zu sein, doch alle diese Ergebnisse waren nicht signifikant. Die Variable ,Alter' verfehlte nur knapp das Signifikanzniveau, während bei anderen Variablen, wie zum Beispiel dem Geschlecht, der Trend nur sehr schwach ausgeprägt war. Auch in unserer Studie bietet das Alter im multivariaten Modell keinen eigenständigen unabhängigen Vorhersagewert (siehe Tabelle 3, Seite 34). Führt man die Berechnung ohne die Variable ,Status der Berufstätigkeit' durch, hat das Alter jedoch einen eigenständigen unabhängigen Vorhersagewert. Dies legt die Vermutung nahe, dass viel Varianz der Variable Alter durch die Variable ,Status der Berufstätigkeit' erklärt wird, da diese vor allem zwischen Berufstätigen und Rentnern trennt.

Wie bereits in Kapitel 2.3.3 (Seite 13 f.) erläutert wurde, konnten aus der Vielzahl von Studien zu den Determinanten von guter Compliance keine allgemeingültigen, konsistenten Erkenntnisse gewonnen werden. Dies gilt insbesondere für soziodemographische Faktoren. Aber auch für therapiespezifische Merkmale gibt es widersprüchliche Forschungsergebnisse. Zum Beispiel fanden Sharkness und Snow (1992) ebenfalls heraus, dass die Compliance bei Patienten, die seit über fünf Jahren Bluthochdruckmedikamente einnahmen, eher höher war als bei Patienten, die erst seit kürzerer Zeit in Behandlung waren. Richardson et al. (1993) hingegen fanden eine Abnahme der Compliance mit der Behandlungsdauer. Unter diesen Einschränkungen müssen die Ergebnisse der 
vorliegenden Studie gesehen werden. Die hier gefundenen Determinanten beschreiben Merkmale von Bluthochdruckpatienten in deutschen Hausarztpraxen. Es scheint allerdings nicht sehr Erfolg versprechend für den behandelnden Arzt zu sein, Aussagen über die Compliance einzelner Patienten nach Vorliegen eines oder mehrerer Charakteristika zu treffen. Die logistische Regression hat gezeigt, dass die Vorhersagewerte der beschriebenen Variablen zwar signifikant, aber dennoch von geringer Aussagekraft sind (siehe Tabelle 3, Seite 34). Damit unterstützt die vorliegende Studie die Einschätzung von Vermeire et al, dass Vorhersagen für die Compliance aufgrund von Patientencharakteristika oder multivariaten Modellen ungenau und fehlerhaft seien (Vermeire et al. 2001).

\subsection{Compliance und Blutdruckeinstellung}

Das interessanteste und am wenigsten erwartete Ergebnis dieser Studie ist sicherlich, dass sich optimal compliante und nicht optimal compliante Studienteilnehmer hinsichtlich der Blutdruckwerte laut Selbstauskunft nicht unterschieden. Die mittleren systolischen Werte waren gleich, die mittleren diastolischen Werte wichen nur um $1 \mathrm{mmHg}$ voneinander ab. Die Rate an Personen mit eingestelltem Blutdruck war in beiden Gruppen nahezu identisch ( $45 \%$ bei Personen mit optimaler Compliance, $44 \%$ bei Personen mit nicht optimaler Compliance). Es stellt sich die Frage, warum die Studienteilnehmer mit besserer Compliance nicht auch bessere Blutdruckwerte erreichten. Zu diskutieren sind in diesem Zusammenhang drei Aspekte: 1. Eine hohe Compliance mit einer Therapie kann nur dann zum Erfolg führen, wenn die Therapie wirksam ist. Könnte die verordnete Medikation wirkungslos gewesen sein, so dass die hohe Compliance mit dieser Therapie keinen Effekt auf den Blutdruck hatte? 2. Wie ist es um die Qualität der angewandten Compliance-Messmethode bestellt; was wurde gemessen? 3 . Welche Aussagekraft haben die auf Selbstauskünften beruhenden Blutdruckwerte?

\subsubsection{Wirksamkeit der Therapie}

Lediglich 40 Studienteilnehmer gaben an, keine blutdrucksenkenden Medikamente zu erhalten. Bei der Berechnung der Complianceraten wurden diese Studienteilnehmer nicht berücksichtigt. Die übrigen 782 Studienteilnehmer, die diesen Fragenkomplex beantworteten, hatten jeweils mindestens ein Antihypertensivum aus der Gruppe der Diuretika, B-Blocker, ACE-Hemmer, AT1- 
Rezeptorblocker oder Calciumkanalblocker im Medikamentenfragebogen angegeben. Es ist unumstritten, dass diese Medikamente bei regelmäßiger Einnahme den Blutdruck senken. Dies wurde in diversen Studien nachgewiesen, von denen hier beispielhaft drei genannt seien (Turnbull et al. 2003; Staessen et. al. 2001; Hansson et al. 1998). Neben der Verschreibung ist naturgemäß auch die Dosierung der Medikamente entscheidend für den Therapieerfolg. Dies haben wir in der vorliegenden Studie nicht ausgewertet, gehen allerdings von einer Gleichverteilung möglicher Unterdosierungen über Patienten mit hoher versus niedriger Compliance aus.

\subsubsection{Erhebung der Compliance}

Zur Erhebung der Compliance gibt es, wie bereits in Kap. 2.3.2 (Seite 10 bis 13) beschrieben, verschiedene Möglichkeiten. So wie sich die Verfahren zur Complianceerhebung unterscheiden, so unterscheiden sich auch die Ergebnisse in ihrer Form. Bei quantitativen Methoden wie dem pill count erhält man als Ergebnis den Quotienten aus eingenommener Medikation zu verschriebener Medikation. Somit ist eine Angabe der Compliance als kontinuierliche Variable in Prozentzahlen möglich. Diese Darstellungsweise wirkt sehr exakt. Allerdings beinhaltet das Ergebnis keine Aussage dazu, nach welchem Muster die Einnahme bzw. das Auslassen der Einnahme erfolgte. So genannte drug holidays (die Einnahme der Medikamente wird für mehrere aufeinander folgende Tage ausgesetzt) werden nicht von sporadisch versäumten Einnahmen unterschieden, obwohl die Einnahmemuster verschiedene Auswirkungen auf den Verlauf des Blutdruckes haben können (Osterberg und Blaschke 2005).

Bei der Verwendung von Patientenfragebögen zur Compliancemessung ist die präzise quantitative Erhebung der eingenommenen Dosen eher unüblich. Hier werden die Antworten zur Compliance in der Regel mit Punkten bewertet. Schließlich wird ein Gesamtscore gebildet, der eine qualitative Aussage zur Compliance des Probanden ermöglichen soll. Die Deutung dieser ComplianceScores ist allerdings nicht immer trivial. Für den Fragebogen nach Hill-Bone haben Krousel-Wood et al. (2005) eine Unterscheidung in optimale und nicht optimale Compliance vorgeschlagen, die auch in dieser Arbeit gewählt wurde. Als optimal compliant galt, wer sämtliche neun Fragen zur Medikamentencompliance mit ,gar nicht' (bzw. ,none of the time') beantwortete und demnach stets alle seine 
Medikamente einnahm. Als nicht optimal compliant galt, wer mindestens eine dieser Fragen abweichend beantwortete. Es wurde nicht zwischen den Studienteilnehmern unterschieden, die nur eine Frage nicht im Sinne optimaler Compliance beantworteten und denjenigen, die mehrere oder alle Fragen nicht im Sinne optimaler Compliance beantworteten. In besonderem Maße diskussionswürdig ist die Einstufung von Studienteilnehmern, die selten die Einnahme ihrer Medikamente versäumten. Für die Blutdruckeinstellung spielt es wahrscheinlich keine entscheidende Rolle, ob eine Person beispielsweise einmal im Monat die Einnahme eines Bluthochdruckmedikaments versäumt, dennoch wäre ein Studienteilnehmer mit diesem Verhalten zur Gruppe mit nicht optimaler Compliance gezählt worden. Es ist somit zweifelhaft, ob die gewählte Grenze zwischen optimaler und nicht optimaler Compliance die Studienteilnehmer hinsichtlich der Relevanz für die Blutdruckeinstellung gut differenziert. Alternativ wäre es möglich gewesen, die Gruppe mit nicht optimaler Compliance weiter zu unterteilen, beispielsweise in eine Gruppe mit mittlerer und eine Gruppe mit schlechter Compliance. Wir haben uns dennoch für die von Krousel-Wood vorgeschlagene Einteilung entschieden, um die Vergleichbarkeit der Ergebnisse zu gewährleisten.

Eine weitere Schwäche von Selbstauskünften bei der Compliancemessung ist natürlich die Gefahr, dass die Antworten in Richtung einer sozialen Erwünschtheit verzerrt werden könnten, so dass das eigentliche Ausmaß der Compliance überschätzt wird (Osterberg und Blaschke 2005). Wenn alle Studienteilnehmer mit gleicher Wahrscheinlichkeit diesem Einfluss unterlägen, wäre allerdings lediglich das Ergebnis der Rate optimaler Compliance betroffen; einen Grund für den fehlenden Unterschied zwischen Patienten mit hoher versus niedriger Compliance bezüglich der Blutdruckwerte wäre damit nicht gegeben.

\subsubsection{Erhebung der Blutdruckwerte}

In der Diagnostik und Therapie der arteriellen Hypertonie nimmt bis heute die Messung des Blutdrucks durch einen Arzt oder medizinisches Fachpersonal in der Arztpraxis oder dem Krankenhaus einen großen Stellenwert ein. Die so erhobenen Werte bilden häufig die Grundlage für die Diagnose, Therapieeinleitung und Therapiesteuerung von Bluthochdruckerkrankungen. Es ist bekannt, dass diese Praxismessungen einige Nachteile haben. So gibt es das Phänomen 
der white-coat hypertension - oder genauer gesagt, in den meisten Fällen isolated office hypertension - aufgrund dessen bei bestimmten Probanden der Blutdruck allein durch die Messung in der Praxis oder im Krankenhaus erhöht ist (Mancia et al. 2007; Pickering et al. 1988; Parati et al. 1998). Umgekehrt gibt es ebenfalls Patienten, bei denen in der Arztpraxis niedrigere Blutdruckwerte gemessen werden als im Tagesdurchschnitt. Dieses Phänomen wird masked hypertension genannt (Ohkubo et al. 2005). Da der Blutdruck über den Tagesverlauf und insbesondere auch nachts schwankt, stellt eine Praxis- oder Klinikmessung nicht immer eine gute Näherung an den ,wahren' Blutdruckwert dar. Als ,wahrer' Blutdruckwert gilt hierbei der über einen längeren Zeitraum gemittelte Blutdruckwert (Pickering et al. 2008). Hier können Langzeitblutdruckmessungen weiterhelfen. Bei dieser Methode wird dem Probanden in der Regel für 24 Stunden ein automatisches Blutdruckmessgerät angelegt, welches in regelmäßigen Intervallen den Blutdruck misst und die Werte speichert. Die Messung kann im Krankenhaus oder im häuslichen Umfeld des Patienten unter Alltagsbedingungen erfolgen. Die gespeicherten Werte werden nach Abschluss der Messung ausgelesen und können als Blutdruckprofil dargestellt werden. Mit dieser Methode und ambulant erhobenen Werten lassen sich hypertoniebedingte Organschäden besser vorhersagen als mit Praxismessungen (Hansen et al. 2006; Mancia et al. 1997). Darüber hinaus bieten Langzeitblutdruckmessungen die Möglichkeit isolated office hypertension und masked hypertension bei Probanden aufzudecken (Ohkubo et al. 2005).

Neben der Blutdruckmessung durch medizinisches Fachpersonal und der Langzeitblutdruckmessung ist es seit Einführung halbautomatischer Blutdruckmessgeräte möglich, dass Patienten ihren Blutdruck im häuslichen Umfeld selbst messen. Die so erhobenen Werte unterliegen nicht dem white-coat-Effekt, sind besser reproduzierbar und sagen hypertoniebedingte Organschäden sowie das Risiko kardiovaskulärer Ereignisse besser voraus als Messungen in medizinischen Einrichtungen (Sega et al. 2005; Fagard et al. 2005; Sakuma et al. 1997; Ohkubo et al. 1998; Verberk et al. 2007). Darüber hinaus gibt es wissenschaftliche Belege, dass anhand von Selbstmessungen hypertoniebedingte Organschäden genau so zuverlässig vorhergesagt werden können wie mittels ambulanter Langzeitmessungen (Stergiou et al. 2007). Dabei gilt, je häufiger gemessen wird, desto besser ist die prognostische Aussagekraft der erhobenen Werte. Jedoch ist 
sie bereits bei geringer Anzahl von Messungen höher als bei Praxismessungen (Ohkubo et al. 2004). Für zuverlässige Selbstmessungen werden an die Geräte bestimmte Anforderungen gestellt. Es sollten Messgeräte mit Oberarmmanschette eingesetzt werden und die Geräte sollten ausreichend validiert sein (O'Brien et al. 2001; Pickering et al. 2008). Neben der validen Blutdruckmessung sind Kostengünstigkeit und leichte Durchführbarkeit Argumente, die Autoren bei ihrer Forderung nach vermehrtem Einsatz von Blutdruckselbstmessungen anführen (Rickerby 2002; Pickering et al. 2008)

Die Höhe des gemessenen Blutdruckwertes ist von Ort und Art der Messung abhängig. Bei Selbstmessungen im häuslichen Umfeld und bei ambulanten 24-Stunden-Messungen werden niedrigere Blutdruckwerte gemessen als bei Messungen durch medizinisches Fachpersonal in medizinischen Einrichtungen (Mancia et al. 1995). Aus diesem Grund gibt es Vorschläge für unterschiedliche Grenzwerte bei der Diagnostik und Therapie der arteriellen Hypertonie (Mancia et al. 2007; Pickering et al. 2008).

Aufgrund des Designs der vorliegenden Studie wurde für die Erhebung der Blutdruckwerte auf Selbstauskünfte der Studienteilnehmer zurückgegriffen. Um valide Blutdruckwerte zu erhalten, sind ambulante Langzeitblutdruckmessungen oder wiederholte Selbstmessungen mit Mittelwertbildung optimale Methoden. Diese waren jedoch in unserer Studie nicht bei jedem eingeschlossenen Patienten zu realisieren. Um die Aussagekraft der Selbstauskünfte besser bewerten zu können, wurden die Teilnehmer deshalb gebeten, neben den Blutdruckwerten anzugeben, wie weit die Messung zurücklag und wer sie durchgeführt hatte. Über die Hälfte der Studienteilnehmer hatten den Blutdruck zuletzt selbst gemessen und ebenfalls bei über der Hälfte der Studienteilnehmer lag die letzte Messung maximal sieben Tage zurück (siehe Tabelle 1, Seite 30). Den Blutdruckwerten unserer Studie liegen also unterschiedliche Messmethoden mit einem großen Anteil Selbstmessungen zugrunde. Es liegt jeweils nur der zuletzt gemessene Wert vor und kein Mittelwert über eine größere Anzahl von Messungen.

Wie sind die von uns erhobenen Blutdruckwerte im Vergleich zu alternativ erhobenen Werten einzuordnen? Sie sind wohl aussagekräftiger als ausschließlich durch medizinisches Fachpersonal in medizinischen Einrichtungen erhobene 
Werte. Wie bereits beschrieben, spiegeln ärztlich durchgeführte Messungen nicht den täglichen Durchschnittsblutdruck des Patienten wider, sondern überschätzen diesen. Patientenselbstmessungen entsprechen eher dem ,wahren' Blutdruck. In der vorliegenden Studie konnte nicht auf gemittelte Werte zurückgegriffen werden. Die erhobenen Werte setzen sich aus Selbstmessungen, ärztlichen Messungen und zu einem geringen Anteil aus Fremdmessungen von anderen Personen zusammen. Die Messungen waren nicht standardisiert. Die Messungen wurden dementsprechend vermutlich $\mathrm{zu}$ verschiedenen Tageszeiten, an unterschiedlichen Wochentagen und in unterschiedlichen häuslichen Situationen durchgeführt. Hierdurch ist wahrscheinlich ein repräsentativeres Bild des alltäglichen Blutdrucks entstanden, als dies durch ausschließlich ärztlich erhobene Blutdruckwerte möglich gewesen wäre.

Für die Interpretation der Ergebnisse dieser Studie ist wichtig, dass Studienteilnehmer mit optimaler Compliance und Studienteilnehmer mit nicht optimaler Compliance sich nicht signifikant bezüglich der Häufigkeit von Selbstmessungen unterschieden. Aufgrund der im Mittel niedrigeren Werte bei Selbstmessungen, wie sie laut Literatur erwartet werden müssten, wäre dies ansonsten ein Ansatzpunkt gewesen, um das überraschende Ergebnis bei der Blutdruckeinstellung beider Gruppen zu erklären.

\subsection{Repräsentativität der Studienteilnehmer}

Für die Beantwortung der Fragestellung war die Auswahl von Personen mit Bluthochdruck in hausärztlicher Behandlung notwendig. Die angewandte Methode erlaubte es, sämtliche Patienten mit der elektronisch dokumentierten Diagnose arterielle Hypertonie, die in den 23 Praxen in Behandlung waren, in den Auswahlprozess einzuschließen. Somit wurden nicht die Personen erfasst, die zwar in hausärztlicher Behandlung waren, deren Bluthochdruck vom Arzt aber nicht erkannt wurde. Da die Compliance mit der medikamentösen Behandlung untersucht wurde und naturgemäß nur die Personen behandelt werden, deren gesundheitliches Problem erkannt wurde, ist diese Tatsache für die Ergebnisse der vorliegenden Arbeit nicht relevant. Die Gefahr, dass bei Patienten fälschlicherweise Bluthochdruck diagnostiziert wurde, scheint eher gering zu sein. In anderen Studien wird zwar die mangelnde Sensitivität der hausärztlichen 
Hypertoniediagnose kritisiert, die Spezifität hingegen nicht (Steckelings et al. 2004).

Im Verlauf des Auswahlverfahrens folgte der manuelle Ausschluss von bestimmten Personen nach vorher festgelegten Kriterien (siehe Anhang 9.1, Seite 58). Durch den Ausschluss von Patienten ohne eindeutig dokumentierte Diagnose einer Bluthochdruckerkrankung und Vertreterpatienten wurde die Gruppe der Studienteilnehmer schärfer eingegrenzt. Bereits verstorbene Patienten wurden ausgeschlossen. Patienten mit eingeschränkter Kommunikationsfähigkeit oder mit psychiatrischen Erkrankungen wie Demenz oder Hirnorganischem Psychosyndrom wurden ausgeschlossen, da eine adäquate Beantwortung der Fragen des Erhebungsinstruments nicht gewährleistet ist. Hinsichtlich der Compliance stellen diese Personengruppen ohnehin eine Besonderheit dar, da die Medikamenteneinnahme meist nicht in eigener Verantwortung der erkrankten Personen, sondern in Händen von Angehörigen und Pflegepersonal liegen dürfte. Patienten mit metastasierten Krebserkrankungen wurden ebenfalls ausgeschlossen. Es wäre ethisch und methodisch nicht korrekt gewesen, diese Patienten über eine Therapie zu befragen, die spätere kardiovaskuläre Folgeerkrankungen verhindern soll, während das Leben der Patienten viel akuter und dramatischer durch eine Krebserkrankung bedroht ist. Wenn die Einnahme antihypertensiver Medikamente in einer solchen Lebenslage möglicherweise in den Hintergrund tritt, dann ist dies nachvollziehbar. Patienten mit der Diagnose „Asthma bronchiale' wurden ausgeschlossen, da diese Patienten bereits in eine zeitgleich durchgeführte Studie der Abteilung Allgemeinmedizin involviert waren. Möglicherweise wurde durch den Ausschluss die Compliance von Patienten mit mehreren chronischen Erkrankungen in der Studie unterrepräsentiert. In der Literatur werden mehrere chronische Erkrankungen und die damit einhergehende Einnahme von mehreren verschiedenen Medikamenten mit niedrigerer Compliance in Verbindung gebracht (Sleight et al. 2006).

Mit 895 Personen, die den Fragebogen beantworteten und an die Abteilung für Allgemeinmedizin zurücksandten, ist die absolute Zahl der Studienteilnehmer recht hoch. Allerdings sind dies nur 26\% der angeschrieben 3443 Personen, die um Teilnahme gebeten wurden. Von vielen Personen (2170; 63\%) erhielten wir gar keine Antwort. Dieser hohe Anteil an Non-Respondern ist für die Interpretation der Ergebnisse von Bedeutung. Bei medizinischen Studien, in 
denen Fragebögen zur Datenerhebung eingesetzt wurden, liegt die durchschnittliche Rücklaufquote bei 60\% (Asch et al. 1997). Nun stellt sich die Frage, weshalb die Rücklaufquote in der vorliegenden Studie so niedrig war. Als Gründe für das Nichtbeantworten von Fragebögen sind in der Literatur unter anderem mangelndes Interesse an der entsprechenden Studie, eine negative Haltung zum Gesundheitssystem, Faulheit oder fehlerhafte Adressen bei der Postzustellung bekannt. In manchen Fällen sind Personen auch nicht in der Lage, Fragebögen zu bearbeiten und zurückzusenden (Tibblin 1965; Janzon et al. 1986; Kotaniemi et al. 2001, Nakash et al. 2008). Neben diesen allgemeinen Gründen kommen in unserem Fall noch einige weitere mögliche hinzu. Der nötige Zeitaufwand, den das Ausfüllen des immerhin achtseitigen Fragebogens in Anspruch nimmt, könnte einige potentielle Teilnehmer abgeschreckt haben. Das Eintragen der eingenommenen Medikamente samt Pharmazentralnummer ist aufwändig, insbesondere wenn Studienteilnehmer viele verschiedene Medikamente einnehmen. So legten einige Studienteilnehmer eine Kopie des Verordnungsplanes ihres Arztes dem Fragebogen bei, um die Angaben nicht per Hand eintragen zu müssen. Ein weiterer Grund könnte darin liegen, dass Personen die sehr persönlichen Daten, wie die eingenommenen Medikamente, aus Datenschutzgründen nicht weitergeben mochten. Andere könnten die Fragen zur Compliance als unangenehme und unangebrachte Kontrolle ihres Verhaltens aufgefasst haben.

Neben der Diskussion über verschiedene Gründe für die niedrige Rate an Respondern ist es für die Interpretation der Ergebnisse wichtig, ob sich die NonResponder in wesentlichen Eigenschaften und Verhaltensweisen bezüglich der Compliance von den Studienteilnehmern unterscheiden. Dies ist letztendlich für die Aussagekraft einer Studie wichtiger als die Rücklaufquote (Asch et al. 1997). Eine Möglichkeit, dieser Frage auf den Grund zu gehen, besteht darin, mit NonRespondern in Kontakt zu treten und sie nochmals um die Beantwortung des Fragebogens zu bitten. In einer Studie über Asthma bronchiale und Rauchverhalten in Finnland wurde auf diese Weise vorgegangen (Kotaniemi et al. 2001). Durch erneuten postalischen und bei Misserfolg telefonischen Kontakt konnten hier knapp die Hälfte der ursprünglichen Non-Responder noch zur Studienteilnahme bewegt werden. In dieser Gruppe waren signifikant mehr Männer, mehr Raucher, mehr selbständig tätige und im Durchschnitt fünf Jahre jüngere Personen als unter den ursprünglichen Studienteilnehmern. Die ehemaligen Non- 
Responder hatten allerdings seltener respiratorische Symptome und litten seltener unter Asthma bronchiale als die Personen, die sich sofort an der Studie beteiligten. In einer weiteren Studie, in der die Rücklaufquote verschiedener Fragebögen untersucht wurde, zeigten sich ebenfalls mehr Männer und eher jüngere Personen unter den Non-Respondern (Hazell et al. 2009).

Für unsere Studie liegen uns keine Informationen über die Gruppe der Non-Responder vor. Es lassen sich nur begründete Vermutungen über die Eigenschaften und die Medikamentencompliance der Personen dieser Gruppe anstellen. Die im vorangegangenen Absatz geschilderten Ergebnisse von Kotaniemi und Hazell legen den Verdacht nahe, dass die Non-Responder unserer Studie eher männlich und eher jünger als die Studienteilnehmer sind. Wenn man die oben genannten Gründe für Nichtbeantwortung von Fragebögen berücksichtigt, wie mangelndes Interesse an der Studie, eine negative Haltung zum Gesundheitssystem, Faulheit oder Unfähigkeit, Fragebögen zu bearbeiten und zurückzusenden, dann könnte man vermuten, dass Personen mit diesen Eigenschaften eher weniger compliant sind als Studienteilnehmer. Ein weiteres Argument für diese These wäre ein durchschnittlich niedrigeres Alter der NonResponder; zeigten sich doch in unserer Studie jüngere Teilnehmer eher weniger compliant als ältere. Somit haben wir möglicherweise in dieser Studie das Ausmaß der optimalen Compliance überschätzt. Es ist weiterhin denkbar, dass zum Beispiel durch einen großen Anteil junger Männer mit niedriger Compliance unter den Non-Respondern die Ergebnisse bezüglich soziodemographischer Charakteristika verfälscht wurden. Das Geschlecht könnte unter diesen Umständen einen signifikanten Zusammenhang mit der Compliance zeigen. Das auffälligste Ergebnis dieser Studie jedoch, die Unabhängigkeit der Blutdruckhöhe von der Medikamentencompliance, wird durch den hohen Anteil an NonRespondern nicht wesentlich berührt. Es bleibt bemerkenswert, dass bei 829 Studienteilnehmern diejenigen mit nicht optimaler Compliance die gleichen Blutdruckwerte wie diejenigen mit optimaler Compliance erreichten.

\subsection{Bedeutung der Ergebnisse und Schlussfolgerung}

Die Rekrutierung von hausärztlichen Patienten ist in Deutschland häufig nicht lückenlos aufzuschlüsseln, vor allem, wenn Hausärzte eigenständig Patienten für eine Studie auswählen und bei der Studienzentrale melden. Die an jedem Punkt 
des Einschlussverfahrens nachvollziehbare Rekrutierung in der vorliegenden Studie ist vor dem Hintergrund epidemiologischer Voraussetzungen als äußerst positiv zu bewerten. Einschränkend muss erwähnt werden, dass die geringe Rücklaufquote die Repräsentativität einiger Ergebnisse hinterfragen läßt. Dies betrifft insbesondere das Ausmaß der Compliance nach Hill-Bone und den Zusammenhang zwischen der Compliance und soziodemographischen bzw. anamnestischen Charakteristika.

In unserer Studie führte eine bessere Compliance mit medikamentöser antihypertensiver Therapie nicht zu niedrigeren Blutdruckwerten. Dieses eher unerwartete Ergebnis muss nicht nur inhaltlich, sondern auch methodisch diskutiert werden. Neben der Frage, inwieweit eine Selbstauskunft der Blutdruckwerte zu verlässlichen Daten führt, muss vor allem die Erhebung der Compliance kritisch beurteilt werden. Die Ergebnisse lassen den Schluss zu, dass das Instrument, so wie es eingesetzt wurde, nicht ausreichend zwischen complianten und non-complianten Personen trennt. Noch einen Schritt weiter gehend, muss die Validität, also die Gültigkeit des Konstrukts der Compliance hinterfragt werden. Einige Anstöße gibt Donner-Banzhoff in einem Artikel über Perspektiven der Hypertoniebehandlung in der hausärztlichen Praxis, in dem er als derzeitige Probleme der hausärztlichen Hypertoniebehandlung unter anderem diskordante Behandlungsziele und professionelle Heteronomie anführt (Donner-Banzhoff 2001). Es sei für eine beiderseits zufrieden stellende Behandlung notwendig, dass sich der gut informierte Patient und sein Arzt auf ein gemeinsames Behandlungsziel einigten. Hierfür müsse der Patient sein individuelles Risiko kennen und gemeinsam mit dem Arzt entscheiden, ob und in welchem Umfang er eine Therapie für sich wünscht. Unter diesen Gesichtspunkten könnte eine sinnvollere Complianceforschung verstärkt den Standpunkt und die Sichtweise der Patienten einbeziehen. Eine Möglichkeit, dies zu verwirklichen, bieten qualitative Patienteninterviews oder Gruppendiskussionen unter Bluthochdruckpatienten, wie sie in einer derzeit laufenden Studie der Abteilung Allgemeinmedizin der Georg-August-Universität Göttingen durchgeführt werden. 


\section{Zusammenfassung}

Hintergrund: Der Bluthochdruck ist ein bedeutender Risikofaktor für Morbidität und Mortalität kardiovaskulärer Erkrankungen. Trotz wirksamer medikamentöser Therapiemöglichkeiten wird eine ausreichende Senkung des Blutdrucks bei vielen Patienten nicht erreicht. Geringe Compliance mit der medikamentösen Therapie wird häufig als eine Ursache hierfür genannt. Patienten mit Bluthochdruck werden vornehmlich in der hausärztlichen Praxis betreut.

Fragestellung: Wie compliant sind hausärztliche Patienten mit Bluthochdruck? Mit welchen demographischen und anamnestischen Parametern hängt die Compliance zusammen? Gibt es einen Zusammenhang zwischen Compliance und Blutdruckeinstellung?

Methode: Aus 23 hausärztlichen Praxen in und um Göttingen wurden mittels elektronisch dokumentierter Behandlungsdaten 6617 Patienten mit der Diagnose ,arterielle Hypertonie' identifiziert. Nach manuellem Ausschluss, vorher festgelegten Kriterien folgend, wurden 3443 Patienten schriftlich um die Studienteilnahme gebeten. 1273 Personen antworteten. Letztendlich sandten 829 Personen den beantworteten Erhebungsbogen zurück und nahmen an der Studie teil. Der Bogen bestand aus Fragen zur Soziodemographie, zur Medikamenteneinnahme, zum eigenen Blutdruck und aus der ,Hill-Bone Compliance to High Blood Pressure Therapy Scale'.

Ergebnisse: Das Durchschnittsalter der Studienteilnehmer betrug 61 Jahre, 51\% waren männlich. Die Rate optimaler Compliance betrug 61\%. Studienteilnehmer mit nicht optimaler Compliance unterschieden sich bezüglich einiger Merkmale von Studienteilnehmern mit optimaler Compliance: Sie waren im Mittel 4 Jahre jünger und häufiger noch berufstätig. Sie lebten seltener mit einem Partner zusammen und nahmen eher erst seit weniger als 5 Jahren Medikamente gegen Bluthochdruck ein. Bezüglich der Blutdruckwerte unterschieden sich beide Gruppen nicht.

Schlussfolgerung: Die nahezu gleichen Blutdruckwerte unter optimal complianten und nicht optimal complianten Studienteilnehmern stellen nicht nur die Eignung des Erhebungsinstrumentes für die valide Bestimmung der Compliance in Frage, sondern lassen auch Zweifel über das hier abgebildete Konstrukt der 
Compliance entstehen. Ein Ausweg aus diesem Erhebungsdilemma könnte eine stärkere Orientierung an der Patientenperspektive sein, die über die Erhebung einer reinen Medikamentenadhärenz hinausgeht. 


\section{Literaturverzeichnis}

Asch DA, Jedrziewski MK, Christakis NA (1997): Response rates to mail surveys published in medical journals. J Clin Epidemiol 50, 1129-1136

Bramley TJ, Gerbino PP, Nightengale BS, Frech-Tamas F (2006): Relationship of blood pressure control to adherence with antihypertensive monotherapy in 13 managed care organizations. J Manag Care Pharm 12, 239-245

Bullinger M, Alonso J, Apolone G, Leplège A, Sullivan M, Wood-Dauphinee $S$, Gandek B, Wagner A, Aaronson N, Bech P, Fukuhara A, Kaasa S, Ware JE (1998): Translating health status questionnaires and evaluating their quality: the IQOLA project approach. J Clin Epidemiol 1ㅗ, 913-923

Burnier M, Schneider MP, Chiolero A, Stubi CL, Brunner HR (2001): Electronic compliance monitoring in resistant hypertension: the basis for rational therapeutic decisions. J Hypertens $\underline{19}$, 335-341

Chalmers I (2006): Why fair tests are needed: a brief history. Evid Based Med $\underline{11}$, 67-68

Cochrane GM (1996): Compliance and outcomes in patients with asthma. Drugs $\underline{52}, 12-19$

Cook CL, Wade WE, Martin BC, Perri M 3rd (2005): Concordance among three self-reported measures of medication adherence and pharmacy refill records. J Am Pharm Assoc 4ㄴ, 151-159

Cramer JA (2002): Effect of partial compliance on cardiovascular medication effectiveness. Heart $\underline{88}, 203-206$

Cramer JA, Mattson RH, Prevey ML, Scheyer RD, Ouellette VL (1989): How often is medication taken as prescribed? A novel assessment technique. JAMA 261, 3273-3277

DiMatteo MR (2004): Variations in patients' adherence to medical recommendations: a quantitative review of 50 years of research. Med Care $\underline{42}, 200-209$

DiMatteo MR, Haskard KB (2006): Further challenges in adherence research: measurements, methodologies, and mental health care. Med Care 444, 297-299

Donner-Banzhoff N (2001): Perspektiven der Hypertoniebehandlung in der hausärztlichen Praxis. Z Arztl Fortbild Qualitatssich $\underline{95}$, 333-338 
Ebell MH, Siwek J, Weiss BD, Woolf SH, Susman JL, Ewigman B, Bowman M (2004): Simplifying the language of evidence to improve patient care: Strength of recommendation taxonomy (SORT): a patient-centered approach to grading evidence in medical literature. J Fam Pract $\underline{53}, 111-120$

Edwards M (2004): Evidence. Lancet 363, 1657

Ezzati M, Hoorn SV, Rodgers A, Lopez AD, Mathers CD, Murray CJ (2003): Estimates of global and regional potential health gains from reducing multiple major risk factors. Lancet $\underline{362}, 271-280$

Fagard RH, Van Den Broeke C, De Cort P (2005): Prognostic significance of blood pressure measured in the office, at home and during ambulatory monitoring in older patients in general practice. J Hum Hypertens $\underline{19}$, 801-807

Garber MC, Nau DP, Erickson SR, Aikens JE, Lawrence JB (2004): The concordance of self-report with other measures of medication adherence: a summary of the literature. Med Care $\underline{42}, 649-652$

Gilbert R, Salanti G, Harden M, See S (2005): Infant sleeping position and the sudden infant death syndrome: systematic review of observational studies and historical review of recommendations from 1940 to 2002. Int J Epidemiol 34, 874887

Glasgow RE, Anderson RM (1999): In diabetes care, moving from compliance to adherence is not enough. Something entirely different is needed. Diabetes Care 22, 2090-2092

Gordis L: Methodologic issues in the measurement of patient compliance; in: Compliance in therapeutic regimens; edit. by Sackett DL, Haynes RB; Johns Hopkins University Press, Baltimore 1976, 51-66

Graubner B: ICD-10-GM 2009. Systematisches Verzeichnis: Internationale statistische Klassifikation der Krankheiten und verwandter Gesundheitsprobleme. 10. Revision - German Modification; Deutscher Ärzte-Verlag, Köln 2008

Hansen TW, Jeppesen J, Rasmussen S, Ibsen H, Torp-Pedersen C (2006): Ambulatory blood pressure monitoring and risk of cardiovascular disease: a population based study. Am J Hypertens $\underline{19}$, 243-250

Hansson L, Zanchetti A, Carruthers SG, Dahlöf B, Elmfeldt D, Julius S, Ménard J, Rahn KH, Wedel H, Westerling S (1998): Effects of intensive blood-pressure lowering and low-dose aspirin in patients with hypertension: principal results of the Hypertension Optimal Treatment (HOT) randomised trial. HOT Study Group. Lancet $\underline{351}$, 1755-1762 
Hasford J, Behrend C, Sangha O: Vergleichende Analyse und Bewertung von Methoden zur Erfassung der Compliance; in: Compliance und Selbstmanagement; hrsg. v. Petermann F; Hogrefe Verlag, Göttingen 1998, 21-44

Haynes RB: Einleitung; in: Compliance Handbuch; edit. by Haynes RB, Taylor DW, Sackett DL; übersetzt und bearbeitet v. Schrey A, Noack EA; Oldenbourg Verlag, München 1982, 11-18

Haynes RB, Taylor DW, Sackett DL, Gibson ES, Bernholz CD, Mukherjee J (1980): Can simple clinical measurements detect patient noncompliance? Hypertension 2, 757-764

Hazell ML, Morris JA, Linehan MF, Frank PI, Frank TL (2009): Factors influencing the response to postal questionnaire surveys about respiratory symptoms. Prim Care Respir J 18, 165-170

Himmel W, Hummers-Pradier E, Kochen MM (2006): Medizinische Versorgung in der hausärztlichen Praxis - Ein neuer Forschungsansatz. Bundesgesundheitsblatt Gesundheitsforschung Gesundheitsschutz 49, 151-159

Hummers-Pradier E, Simmenroth-Nayda A, Scheidt-Nave C, Scheer N, Fischer T, Niebling W, Kochen MM (2003): Versorgungsforschung mit hausärztlichen Routinedaten - Sind Behandlungsdatenträger-(BDT-)Exporte geeignet? Gesundheitswesen $\underline{65}, 109-114$

Janzon L, Hanson BS, Isacsson SO, Lindell SE, Steen B (1986): Factors influencing participation in health surveys. Results from prospective population study 'Men born in 1914' in Malmö, Sweden. J Epidemiol Community Health $\underline{40}$, 174-177

Johnson BF, Whelton A (1994): A study design for comparing the effects of missing daily doses of antihypertensive drugs. Am J Ther $\underline{1}, 260-267$

Kearney PM, Whelton M, Reynolds K, Muntner P, Whelton PK, He J (2005): Global burden of hypertension: analysis of worldwide data. Lancet $\underline{365}, 217-223$

Kim MT, Hill MN, Bone LR, Levine DM (2000): Development and testing of the Hill-Bone Compliance to High Blood Pressure Therapy Scale. Prog Cardiovasc Nurs $\underline{15}, 90-96$

Kjellgren KI, Ahlner J, Saljo R (1995): Taking antihypertensive medication controlling or co-operating with patients? Int J Cardiol 47, 257-268 
Kotaniemi JT, Hassi J, Kataja M, Jönsson E, Laitinen LA, Sovijärvi AR, Lundbäck B (2001): Does non-responder bias have a significant effect on the results in a postal questionnaire study? Eur J Epidemiol 17, 809-817

Kravitz RL, Melnikow J (2004): Medical adherence research: time for a change in direction? Med Care 42, 197-199

Kreienbrock L, Schach S: Epidemiologische Methoden. Gustav Fischer Verlag, Stuttgart 1995

Krousel-Wood M, Muntner P, Jannu A, Desalvo K, Re RN (2005): Reliability of a medication adherence measure in an outpatient setting. Am J Med Sci 330, 128133

Mancia G, Sega R, Bravi C, De Vito G, Valagussa F, Cesana G, Zanchetti A (1995): Ambulatory blood pressure normality: results from the PAMELA study. J Hypertens $\underline{13}, 1377-1390$

Mancia G, Zanchetti A, Agabiti-Rosei E, Benemio G, De Cesaris R, Fogari R, Pessina A, Porcellati C, Rappelli A, Salvetti A, Trimarco B (1997): Ambulatory blood pressure is superior to clinic blood pressure in predicting treatment-induced regression of left ventricular hypertrophy. SAMPLE Study Group. Study on Ambulatory Monitoring of Blood Pressure and Lisinopril Evaluation. Circulation $\underline{95}, 1464-1470$

Mancia G, De Backer G, Dominiczak A, Cifkova R, Fagard R, Germano G, Grassi G, Heagerty AM, Kjeldsen SE, Laurent S, Narkiewicz K, Ruilope L, Rynkiewicz A, Schmieder RE, Boudier HA, Zanchetti A (2007): 2007 guidelines for the management of arterial hypertension: the task force for the management of arterial hypertension of the European Society of Hypertension (ESH) and of the European Society of Cardiology (ESC). J Hypertens 25, 1105-1187

McPherson K (2004): Where are we now with hormone replacement therapy? $\mathrm{BMJ}$ 328, 357-358

Morisky DE, Green LW, Levine DM (1986): Concurrent and predictive validity of a self-reported measure of medication adherence. Med Care 24, 67-74

Nakash RA, Hutton JL, Lamb SE, Gates S, Fisher J (2008): Response and nonresponse to postal questionnaire follow-up in a clinical trial - a qualitative study of the patient's perspective. J Eval Clin Pract 14, 226-235

O'Brien E, Waeber B, Parati G, Staessen J, Myers MG (2001): Blood pressure measuring devices: recommendations of the European Society of Hypertension. BMJ $\underline{322}, 531-536$ 
Ohkubo T, Imai Y, Tsuji I, Nagai K, Kato J, Kikuchi N, Nishiyama A, Aihara A, Sekino M, Kikuya M, Ito S, Satoh H, Hisamichi S (1998): Home blood pressure measurement has a stronger predictive power for mortality than does screening blood pressure measurement: a population-based observation in Ohasama, Japan. J Hypertens $\underline{16}$, 971-975

Ohkubo T, Asayama K, Kikuya M, Metoki H, Hoshi H, Hashimoto J, Totsune K, Satoh H, Imai Y (2004): How many times should blood pressure be measured at home for better prediction of stroke risk? Ten-year follow-up results from the Ohasama study. J Hypertens 22, 1099-1104

Ohkubo T, Kikuya M, Metoki H, Asayama K, Obara T, Hashimoto J, Totsune K, Hoshi H, Satoh H, Imai Y (2005): Prognosis of masked hypertension and white-coat hypertension detected by 24-h ambulatory blood pressure monitoring. J Am Coll Cardiol 46, 508-515

Osterberg L, Blaschke T (2005): Adherence to medication. N Engl J Med $\underline{353}$, 487-497

Parati G, Ulian L, Santucci C, Omboni S, Mancia G (1998): Difference between clinic and daytime blood pressure is not a measure of the white coat effect. Hypertension 31, 1185-1189

Paterson DL, Potoski B, Capitano B (2002): Measurement of adherence to antiretroviral medications. J Acquir Immune Defic Syndr 31, 103-106

Pickering TG, James GD, Boddie C, Harshfield GA, Blank S, Laragh JH (1988): How common is white coat hypertension? JAMA 259, 225-228

Pickering TG, Miller NH, Ogedegbe G, Krakoff LR, Artinian NT, Goff D (2008): Call to action on use and reimbursement for home blood pressure monitoring: a joint scientific statement from the American Heart Association, American Society Of Hypertension, and Preventive Cardiovascular Nurses Association. Hypertension $\underline{52}, 10-29$

Psychrembel W: Psychrembel Klinisches Wörterbuch. De Gruyter, Berlin 2004

Pullar T, Kumar S, Tindall H, Feely M (1989): Time to stop counting the tablets? Clin Pharmacol Ther $\underline{46}$, 163-168

Rangno RE, Langlois S (1982): Comparison of withdrawal phenomena after propranolol, metoprolol and pindolol. Br J Clin Pharmacol $\underline{13}$, 345-351 
Richardson MA, Simons-Morton B, Annegers JF (1993): Effect of perceived barriers on compliance with antihypertensive medication. Health Educ Q 20, 489503

Rickerby J (2002): The role of home blood pressure measurement in managing hypertension: an evidence-based review. J Hum Hypertens $\underline{16}$, 469-472

Roche Lexikon Medizin. 5. Auflage; Urban \& Fischer, München 2003

Rudd P (1979): In search of the gold standard for compliance measurement. Arch Intern Med 139, 627-628

Rudd P, Byyny RL, Zachary V, LoVerde ME, Mitchell WD, Titus C, Marshall G (1988): Pill count measures of compliance in a drug trial: variability and suitability. Am J Hypertens $\underline{1}$, 309-312

Sackett DL: Introduction; in: Compliance with therapeutic regimens; hrsg. v. Sackett DL, Haynes RB; Johns Hopkins University Press, Baltimore 1976, 1-6

Sackett DL, Rosenberg WM, Gray JA, Haynes RB, Richardson WS (1996): Evidence based medicine: what it is and what it isn't. BMJ $\underline{312}, 71-72$

Sakuma M, Imai Y, Nagai K, Watanabe N, Sakuma H, Minami N, Satoh H, Abe K (1997): Reproducibility of home blood pressure measurements over a 1-year period. Am J Hypertens $\underline{10}, 798-803$

Schroeder K, Fahey T, Ebrahim S (2004): How can we improve adherence to blood pressure-lowering medication in ambulatory care? Systematic review of randomized controlled trials. Arch Intern Med 164, 722-732

Sega R, Facchetti R, Bombelli M, Cesana G, Corrao G, Grassi G, Mancia G (2005): Prognostic value of ambulatory and home blood pressures compared with office blood pressure in the general population: follow-up results from the Pressioni Arteriose Monitorate e Loro Associazioni (PAMELA) study. Circulation $\underline{111}, 1777-1783$

Sharkness CM, Snow DA (1992): The patient's view of hypertension and compliance. Am J Prev Med 모, 141-146

Sleight P, Pouleur H, Zannad F (2006): Benefits, challenges, and registerability of the polypill. Eur Heart J 27, 1651-1656

Staessen JA, Wang JG, Thijs L (2001): Cardiovascular protection and blood pressure reduction: a meta-analysis. Lancet $\underline{358}, 1305-1315$ 
Steckelings UM, Stoppelhaar M, Sharma AM, Wittchen HU, Krause P, Kupper B, Kirch W, Pittrow D, Ritz E, Goke B, Lehnert H, Tschope D, Hofler M, Pfister H, Unger T (2004): HYDRA: possible determinants of unsatisfactory hypertension control in German primary care patients. Blood Press $\underline{13}, 80-88$

Steiner JF, Earnest MA (2000): The language of medication-taking. Ann Intern Med 132, 926-930

Stergiou GS, Argyraki KK, Moyssaki I, Mastorantonakis SE, Achimastos AD, Karamanos VG, Roussias LG (2007): Home blood pressure is as reliable as ambulatory blood pressure in predicting target-organ damage in hypertension. Am J Hypertens 므, 616-621

Thamm M (1999): Blutdruck in Deutschland - Zustandsbeschreibung und Trends. Gesundheitswesen $\underline{61}$, 90-93

Tibblin G (1965): A population study of 50-year-old men. An analysis of the nonparticipation group. Acta Med Scand 178, 453-459

Turnbull F; Blood Pressure Lowering Treatment Trialists' Collaboration (2003): Effects of different blood-pressure-lowering regimens on major cardiovascular events: results of prospectively-designed overviews of randomised trials. Lancet 362, 1527-1535

Verberk WJ, Kroon AA, Jongen-Vancraybex HA, de Leeuw PW (2007): The applicability of home blood pressure measurement in clinical practice: a review of literature. Vasc Health Risk Manag $\underline{3}$, 959-966

Vermeire E, Hearnshaw H, Van Royen P, Denekens J (2001): Patient adherence to treatment: three decades of research. A comprehensive review. J Clin Pharm Ther $\underline{26}, 331-342$

Walsh JC, Mandalia S, Gazzard BG (2002): Responses to a 1 month self-report on adherence to antiretroviral therapy are consistent with electronic data and virological treatment outcome. AIDS $\underline{16}, 269-277$

Weiss M, Britten N (2003): What is concordance? Pharm J 271, 493

Wetzels G, Nelemans P, van Wijk B, Broers N, Schouten J, Prins M (2006): Determinants of poor adherence in hypertensive patients: development and validation of the "Maastricht Utrecht Adherence in Hypertension (MUAH)questionnaire". Patient Educ Couns $\underline{64}, 151-158$ 
Wolf-Maier K, Cooper RS, Banegas JR, Giampaoli S, Hense HW, Joffres M, Kastarinen M, Poulter N, Primatesta P, Rodriguez-Artalejo F, Stegmayr B, Thamm M, Tuomilehto J, Vanuzzo D, Vescio F (2003): Hypertension prevalence and blood pressure levels in 6 European countries, Canada, and the United States. JAMA 289, 2363-2369

Wolf-Maier K, Cooper RS, Kramer H, Banegas JR, Giampaoli S, Joffres MR, Poulter N, Primatesta P, Stegmayr B, Thamm M (2004): Hypertension treatment and control in five European countries, Canada, and the United States.

Hypertension $\underline{43}, 10-17$ 


\section{Anhang}

\subsection{Ausschlusskriterien}

1. Keines; Patient(in) wird angeschrieben

MED'IIP

Medizinische

Versorgung

in der Praxis

(Bei eindeutig dokumentierter Diagnose „primäre arterielle Hypertonie“ oder „Belastungshypertonie")

2. Keine oder keine eindeutig dokumentierte Diagnose „primäre arterielle Hypertonie“

(Dazu gehören: Verdacht auf Hypertonie ; Grenzwerthypertonie; labile Hypertonie; sekundäre Hypertonie, auch medikamenteninduziert auf der Basis einer anderen Erkrankung, z.B. bei Cortisondauertherapie; muskulärer Hypertonus; 115.; 127.)

3. Notfall-, Vertreterpatient(in); kein(e) Stammpatient(in)

4. Wechsel der Hausärztin/ des Hausarztes; Verzogen

5. Verstorben

6. Eingeschränkte Kommunikationsfähigkeit (Analphabetismus, geringe Deutschkenntnisse, Aphasie, Alexie, Narkolepsie)

7. Bestimmte psychiatrische Erkrankungen

(Dazu gehören: Demenz; Psychose; andere Verwirrtheitszustände; geistige Behinderung; Alzheimer; HOPS; schwerer Drogen-, Alkohol-, Medikamentenabusus - nach Rücksprache mit der Praxismitarbeiterin)

8. Pflegebedürftigkeit oder Immobilität

(Dazu gehören: gesetzliche Betreuung; Hausbesuche; Dekubitus; Stuhlinkontinenz; dokumentierte Verschreibung von Windelhosen, Liegeunterlagen, eines Toilettenstuhls; Rollstuhl)

9. Komorbiditäten:

Metastasierte Krebserkrankungen

10. Sonstige Gründe, die eine Studienteilnahme unmöglich machen. Bitte Gründe angeben!

11. Asthma bronchiale (Dadurch werden Überschneidungen mit dem A2-Projekt verhindert. In Absprache mit der Studienleitung des A2-Projektes werden nach Durchsicht der BDT-Daten darüber hinaus die PatientInnen ausgeschlossen, die aufgrund eines Verdachts auf Asthma bronchiale in das A2Projekt eingeschlossen werden.) 
Erläuterungen zu den Ausschlusskriterien:

- Alle PatientInnen, die eindeutig eingeschlossen werden können, werden mit dem Code "1" versehen.

- Alle PatientInnen, bei denen nach Durchsicht der BDT-Daten Klärungsbedarf bzgl. der Ausschlusskriterien besteht, erhalten zunächst den Code „1“ und die entsprechende Anmerkung, die mit der Praxismitarbeiterin zu klären ist (z.B. schwerer Alkoholmissbrauch?, NotfallpatientIn? etc.).

- Alle PatientInnen, die eindeutig ausgeschlossen werden können, erhalten den entsprechenden Code; sollten bei einer Patientin/ einem Patienten mehrere Ausschlusskriterien zugleich bestehen, gilt folgende Priorität:

o 11 - Asthma bronchiale; Verdacht auf Asthma bronchiale, nach Rücksprache mit der Leitung des A2-Projektes

o 2 - Keine oder keine eindeutig dokumentierte Diagnose „primäre arterielle Hypertonie"

o 3 - Notfall-, VertreterpatientIn

o 4-Wechsel der Hausärztin/ des Hausarztes; Verzogen

o 5 - Verstorben

o 6 - eingeschränkte Kommunikationsfähigkeit

o 8 -Pflegebedürftigkeit/ Immobilität

o 7 - bestimmte psychiatrische Erkrankungen

o 9 - Komorbiditäten 


\subsection{Anschreiben an die Patienten vom Hausarzt}

Dr. med. Max Mustermann

Neue Straße 22

Facharzt für Allgemeinmedizin

37186 Moringen

\section{Liebe Patientin, lieber Patient,}

als Ihr Hausarzt bitte ich Sie, an einer Studie

der Universität Göttingen teilzunehmen.

Die Abteilung Allgemeinmedizin führt eine Untersuchung zur Behandlung des Bluthochdrucks durch. Viele Patientinnen und Patienten, die unter Bluthochdruck leiden, kommen sehr gut mit Ihren Medikamenten zurecht. Es gibt aber auch andere, die zweifeln, ob sie Medikamente zur Behandlung einnehmen sollten und daher manchmal auch nach anderen Möglichkeiten suchen, den Bluthochdruck in den Griff zu bekommen. In der Vergangenheit hat die medizinische Forschung diese Entscheidungen und Überlegungen von Patientinnen und Patienten, häufig nicht beachtet. Die Abteilung Allgemeinmedizin hat es sich nun zur Aufgabe gemacht, Patientenentscheidungen zu untersuchen, um von Patientinnen und Patienten zu lernen und sie in Zukunft in ihrem Entscheidungsprozess besser unterstützen zu können.

Interessierte Patientinnen und Patienten erhalten per Post von der Abteilung Allgemeinmedizin zunächst einen Fragebogen, und werden gebeten diesen auszufüllen. Im weiteren Verlauf der Studie werden die Studienteilnehmerinnen und -teilnehmer angerufen; in einem 10minütigen Telefoninterview wird die Studienassistentin sich dann mit innen über ihre Behandlung des Bluthochdrucks unterhalten.

Wenn Sie Interesse haben, an der Studie teilzunehmen, senden Sie bitte einfach folgenden Antwortbogen in dem beigelegten Briefumschlag zurück. Die Gebühr bezahlt die Universität! Geben Sie bitte unbedingt Ihren Namen, Ihre Adresse und Telefonnummer an, da die Mitarbeiterinnen der Universität sonst nicht mit Ihnen in Kontakt treten können.

Eine Teilnahme an der Studie hat keinen Einfluss auf Ihre weitere hausärztliche Behandlung. Sie können jederzeit von der Teilnahme zurücktreten, ohne dass sich dies auf Ihre Behandlung auswirkt. Falls Sie in der Vergangenheit bereits an einer anderen Studie teilgenommen haben, ist dies KEIN Hinderungsgrund für eine erneute Teilnahme!

Für die Studienleitung ist es von großem Nutzen, dass Sie den Bogen auch dann ausfüllen, wenn Sie kein Interesse haben, an der Studie teilzunehmen oder aus anderen Gründen nicht teilnehmen wollen oder können. Eine Angabe von Name und Adresse ist dann natürlich nicht nötig.

Wenn Sie Fragen zur Studie haben, können Sie in der Abteilung Allgemeinmedizin der Universität anrufen. Die Telefonnummern finden Sie auf dem Antwortbogen.

Mit herzlichen Grüßen

Dr. med. Max Mustermann 
Georg- August- Universität Göttingen • Bereich Humanmedizin Universitätsklinikum • Medizinische Fakultät

\section{Abteilung Allgemeinmedizin}

Direktor: Univ.-Prof. Dr. med. Michael M. Kochen, MPH, FRCGP

Humboldtallee 38 • 37073 Göttingen

\section{Antwortbogen}

\section{"Compliance bei der Behandlung des Bluthochdrucks"}

Bitte füllen Sie folgenden Bogen aus und senden ihn in dem beiliegenden frankierten und adressierten Umschlag zurück. Ein Zerteilen des Bogens ist nicht notwendig.

Wenn Sie teilnehmen möchten, machen Sie bitte hier ein Kreuz und füllen die nachfolgenden Felder aus.

\section{“ Ja, ich möchte an der oben genannten Studie teilnehmen.}

Bitte füllen Sie folgende Felder aus. Indem Sie uns diesem Bogen zurückschicken, erklären Sie sich einverstanden, dass die Abteilung Allgemeinmedizin Ihnen einen Fragebogen zuschickt. Ein Rücktritt ist danach jederzeit ohne Angaben von Gründen möglich.

Nachname:

Vorname:

Geburtsdatum:

Straße u. Hausnr.:

PLZ und Ort:

Telefonnummer:

Günstigste Zeit

für einen Rückruf:

Wenn Sie nicht teilnehmen möchten, machen Sie bitte hier ein Kreuz und füllen die nachfolgenden Felder aus.

“ Nein, eine Studienteilnahme kommt für mich nicht in Frage.

Bitte füllen Sie dennoch folgende Felder aus! Rückschlüsse auf Ihre Person sind anhand der Daten NICHT möglich! Ich bin am geboren und " weiblich

“ männlich.

Grund für die Absage (freiwillige Angabe):

Wenn Sie Fragen haben, rufen Sie uns gerne unter den unten angegebenen Telefonnummern an. Vielen Dank! 


\subsection{Aufklärung und Einverständniserklärung}

Georg- August- Universität Göttingen • Bereich Humanmedizin

Universitätsklinikum • Medizinische Fakultät

\section{Abteilung Allgemeinmedizin}

Direktor: Univ.-Prof. Dr. med. Michael M. Kochen, MPH, FRCGP

Anrede

Allgemeinmedizin, Postfach 3742/43, 37070 Göttingen

Vorname Name

Straße Nr.

PLZ Ort

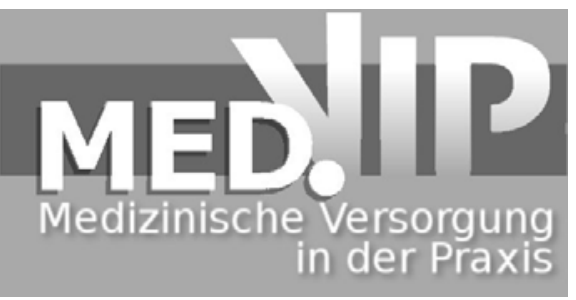

Göttingen, den 21. Oktober 2009

Sehr geehrte(r) Serienbrieffunktion,

von Ihrer Hausärztin Frau / Herrn Hausarzt wurden Sie zur Teilnahme an der Studie „Compliance bei der Behandlung des Bluthochdrucks" der Abteilung Allgemeinmedizin der Universität Göttingen eingeladen. Für Ihre Teilnahmebereitschaft bedanken wir uns herzlich.

Mit diesem Anschreiben überreichen wir Ihnen folgendes Studienmaterial:

- einen Bogen mit Informationen zum Studienablauf (Aufklärung zur Teilnahme an der Studie),

- einen 8-seitigen Fragebogen zu verschiedenen Themengebieten,

- zwei Exemplare der Einverständniserklärung,

- einen freigestempelten und adressierten Rückumschlag.

Bitte senden Sie

- den ausgefüllten Fragebogen und

- eines der beiden Exemplare der Einverständniserklärung mit Ihrer Unterschrift

so bald wie möglich in dem beigelegten Rückumschlag an uns zurück (Gebühr bezahlt die Universität!). Falls Sie Fragen haben, können Sie uns gerne unter den unten angegebenen Telefonnummern anrufen.

Mit freundlichen Grüßen

Gabriella Marx, MA

Studienleiterin

Tel. (0551) 39-9250
Hannelore Schneider-Rudt

Studienassistentin

Tel. (0551) 39-14228 
Georg- August- Universität Göttingen • Bereich Humanmedizin Universitätsklinikum • Medizinische Fakultät

\title{
Abteilung Allgemeinmedizin
}

Direktor: Univ.-Prof. Dr. med. Michael M. Kochen, MPH, FRCGP Humboldtallee 38 • 37073 Göttingen

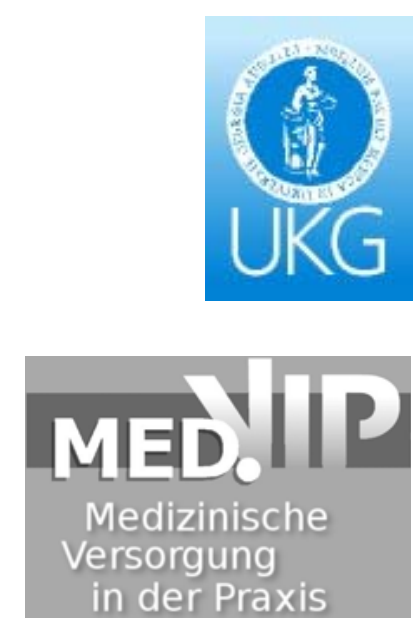

\section{Aufklärung zur Teilnahme an der Studie „Compliance bei der Behandlung des Bluthochdrucks"}

\begin{abstract}
Liebe Teilnehmerin,
\end{abstract}
lieber Teilnehmer,

die Abteilung Allgemeinmedizin der Universität Göttingen führt diese wissenschaftliche Studie durch, um mehr über mögliche Probleme bei der hausärztlichen Behandlung des Bluthochdrucks zu erfahren.

Mit diesem Schreiben haben Sie von uns das Studienmaterial erhalten. Nachdem Sie die Unterlagen an uns zurückgeschickt haben, werden Ihre Angaben von den Mitarbeiterinnen und Mitarbeitern der Universität unter Berücksichtung der Datenschutzbestimmungen ausgewertet. Im Anschluss daran, nach etwa ein bis zwei Wochen, wird eine Mitarbeiterin der Abteilung Allgemeinmedizin sich mit einigen Studienteilnehmerinnen und Studienteilnehmern erneut in Verbindung setzen. Die Auswahl erfolgt per Zufall. In einem kurzen Telefongespräch werden Sie dann gebeten, ein paar Fragen zur Behandlung Ihres Bluthochdrucks zu beantworten.

Die Teilnahme an dieser Studie ist freiwillig, eine Nichtteilnahme bringt keinerlei Nachteile mit sich. Ihre Daten werden ausschließlich für Studienzwecke gespeichert, eine Weitergabe an Dritte, z.B. Ihre Hausärztin oder Ihren Hausarzt, ist ausgeschlossen. Sie können Ihr einmal gegebenes Einverständnis jederzeit ohne Angabe von Gründen zurückziehen, ohne dass Ihnen dadurch irgendwelche Nachteile entstehen; alle bis dahin erhobenen Daten würden in diesem Falle vernichtet werden.

Mit freundlichen Grüßen

Gabriella Marx, MA

Studienleiterin

Tel. (0551) 39-9250
Hannelore Schneider-Rudt

Studienassistentin

Tel: (0551) 39-14228 
Georg- August- Universität Göttingen • Bereich Humanmedizin Universitätsklinikum • Medizinische Fakultät

\section{Abteilung Allgemeinmedizin}

Direktor: Univ.-Prof. Dr. med. Michael M. Kochen, MPH, FRCGP Humboldtallee 38 • 37073 Göttingen

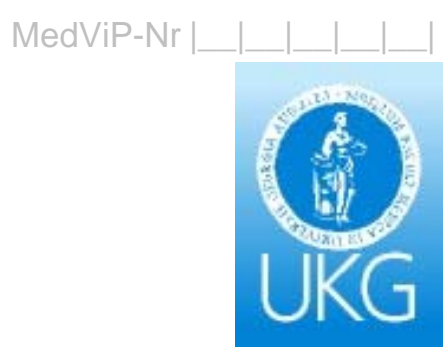

\section{Einverständniserklärung für die Teilnahme an der Studie} "Compliance bei der Behandlung des Bluthochdrucks"

Ich, , wurde vollständig über die Studie mit dem o.g. Titel aufgeklärt. Ich habe den Aufklärungstext gelesen und verstanden. Ich hatte die Möglichkeit, Fragen zu stellen und habe die Antworten verstanden und akzeptiere sie.

Ich hatte ausreichend Zeit, mich für die Teilnahme an dieser Studie zu entscheiden und weiß, dass die Teilnahme freiwillig und mit keinerlei persönlichem Nutzen für mich verbunden ist. Ich weiß, dass ich jederzeit und ohne Angaben von Gründen diese Zustimmung widerrufen kann, ohne dass durch diesen Entschluss Nachteile für mich entstehen. Die bisher erhobenen Daten würden in diesem Falle vernichtet werden. Ich weiß, dass meine Teilnahme oder ein möglicher Rücktritt von der Teilnahme keinerlei Einfluss auf die Behandlung durch meine Hausärztin oder meinen Hausarzt haben. Ich bin damit einverstanden, dass die im Rahmen der Studie erhobenen Daten in anonymisierter Form gespeichert und zu Forschungszwecken verwendet werden.

Ich habe die Aufklärung zur Teilnahme an der Studie und eine Kopie der Einverständniserklärung erhalten. 


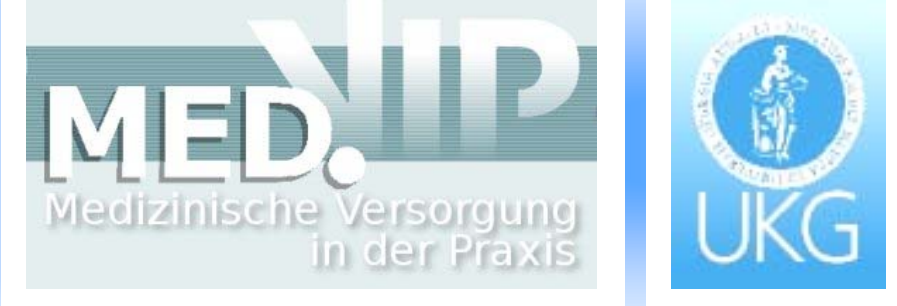

\section{Liebe Studienteilnehmerin, lieber Studienteilnehmer,}

dies ist der Fragebogen zur Studie "Compliance bei der Behandlung des Bluthochdrucks" der Abteilung Allgemeinmedizin der Universität Göttingen. Er enthält Fragen zu Medikamenten und deren Einnahme, zu Ihrem Bluthochdruck sowie zu Ihrer Person.

Selbstverständlich ist die Beantwortung freiwillig und ohne jeden Einfluss auf Ihre Behandlung. Alle Ihre Angaben werden nach den Bestimmungen des Datenschutzes anonym ausgewertet, sodass keinerlei Rückschlüsse auf Ihre Person möglich sind. Eine Weitergabe an Dritte ist ausgeschlossen.

\section{Bitte beachten Sie beim Ausfüllen Folgendes:}

- Beantworten Sie bitte alle Fragen vollständig mit jeweils einem Kreuz. Sollten Sie bei der Beantwortung einer Frage nicht sicher sein, kreuzen Sie die Antwort an, die am ehesten auf Sie zutrifft.

- Bei der Beantwortung können Sie sich ruhig Zeit nehmen, sollten jedoch nicht ins Grübeln geraten, sondern eher spontan antworten.

- Wichtig ist, dass Sie die Fragen selbst beantworten und sich dabei nicht „helfen“ oder gar „vertreten“ lassen.

\section{Vielen Dank für Ihre Mitarbeit!}

Ihr MedViP-Team

$\begin{aligned} \text { Gabriella Marx, MA } & \text { Hannelore Schneider-Rudt } \\ \text { Studienleiterin } & \text { Studienassistentin } \\ \text { Tel. (0551) 39-9250 } & \text { Tel. (0551) 39-14228 }\end{aligned}$


Auf den folgenden zwei Seiten geht es um die Einnahme von Medikamenten.

Haben Sie innerhalb der letzten 7 Tage Medikamente eingenommen?

Ja $\square 2$ Nein

Wenn ja, welche Medikamente waren das? Machen Sie bitte Ihre Angaben auf der nächsten Seite entsprechend der unten geschilderten Vorgehensweise! Bitte tragen Sie auch Medikamente ein, die Sie nicht auf Rezept von der Ärztin/vom Arzt verordnet bekommen haben (z.B. frei verkäufliche Schmerzmittel).

Gehen Sie bitte folgendermaßen vor:

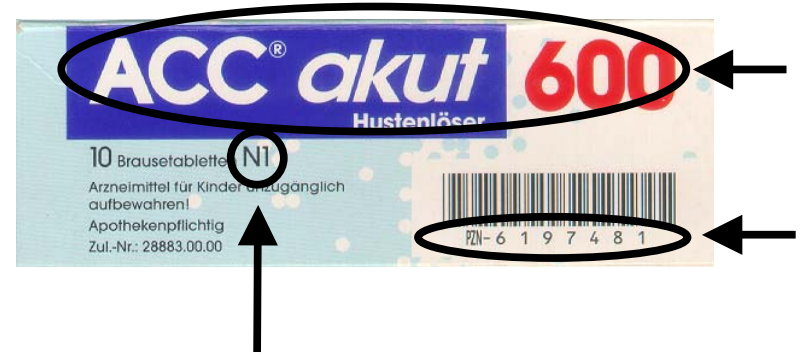

Wenn vorhanden: Kreuzen Sie die Geben Sie den genauen und vollständigen Namen des Medikamentes an.

Wenn vorhanden: Tragen Sie die PZN-Nummer ein. Sie finden Sie meist unter dem Strichcode auf einer der sechs Seiten der Medikamentenschachtel. Die Buchstabenfolge „PZN“ steht immer vor der Nummer; die Nummer ist immer 7-stellig.

Normpackungsgröße an. Die Normpackungsgröße ist meistens vor oder hinter den Angaben zur Packungsgröße als N1, N2 oder N3 angegeben.

Tragen Sie bitte für jedes Medikament auch noch Folgendes ein:

- die Darreichungsform (z.B. Dragees, Tabletten, Kapseln, Tropfen, Hübe)

- die empfohlene Dosis zur regelmäßigen Einnahme (z.B. 3x täglich 1 Tabl., darzustellen als 1-1-1) oder zur Einnahme nach Bedarf (z.B. bei akuten Beschwerden)

- ob es Ihnen vom Arzt auf Rezept verordnet wurde oder nicht.

Für das zuvor abgebildete Medikament zeigen wir Ihnen, wie Sie die Einträge für Ihre eigenen Medikamente vornehmen sollten:

$$
A|C| C|A| K|U| T|\quad 6| 0 \mid 0
$$

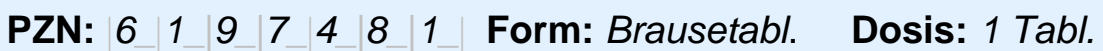
Grund für Einnahme: zum Schleimlösen

\begin{tabular}{|c|cc|cc|}
\cline { 2 - 4 } & \multicolumn{2}{|c|}{$\begin{array}{c}\text { Verordnung } \\
\text { der } \\
\text { Packungs } \\
\text { größe }\end{array}$} & \multicolumn{2}{c|}{$\begin{array}{c}\text { vom Arzt } \\
\text { Einnahme } \\
\text { Regel }\end{array}$} \\
mäßig & Bedart & Ja & Nein \\
\hline N1 & & & & \\
$\square N 2$ & $\square$ & $\square$ & $\square$ & $\square$ \\
$\square N 3$ & & & & \\
\hline
\end{tabular}




\begin{tabular}{|c|c|c|c|c|c|}
\hline Bitte fahren Sie fort: & $\begin{array}{l}\text { Packungs } \\
\text { größe }\end{array}$ & $\begin{array}{l}\text { Verorc } \\
\text { d } \\
\text { Einna } \\
\text { Regel } \\
\text { mäßig }\end{array}$ & \begin{tabular}{l|} 
dnung \\
er \\
ahme \\
Nach \\
Bedarf
\end{tabular} & $\begin{array}{l}\text { vom } \\
\text { vero } \\
\mathrm{Ja}\end{array}$ & $\begin{array}{l}\text { Arzt } \\
\text { rdnet } \\
\text { Nein }\end{array}$ \\
\hline 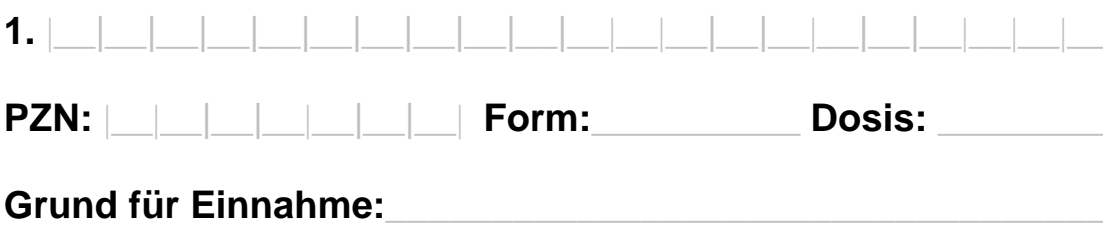 & $\begin{array}{l}\square \mathrm{N} 1 \\
\square \mathrm{N} 2 \\
\square \mathrm{N3}\end{array}$ & $\square$ & $\square$ & $\square$ & $\square$ \\
\hline 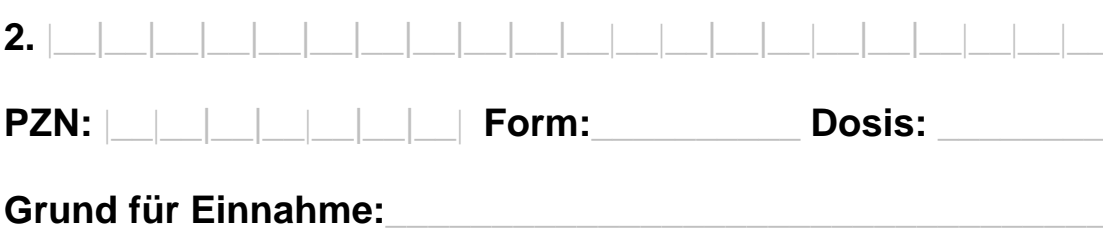 & $\begin{array}{l}\square \mathrm{N} 1 \\
\square \mathrm{N} 2 \\
\square \mathrm{N3}\end{array}$ & $\square$ & $\square$ & $\square$ & $\square$ \\
\hline 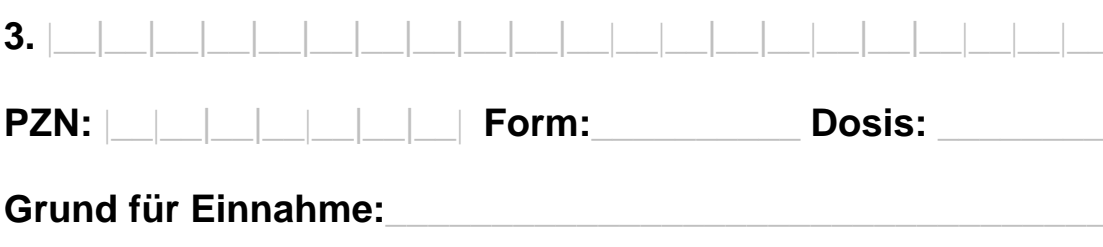 & $\begin{array}{l}\square \mathrm{N} 1 \\
\square \mathrm{N} 2 \\
\square \mathrm{N3}\end{array}$ & $\square$ & $\square$ & $\square$ & $\square$ \\
\hline 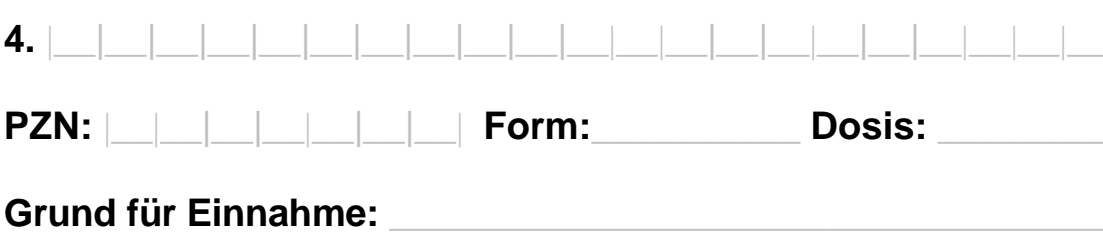 & $\begin{array}{l}\square \mathrm{N} 1 \\
\square \mathrm{N} 2 \\
\square \mathrm{N3}\end{array}$ & $\square$ & $\square$ & $\square$ & $\square$ \\
\hline 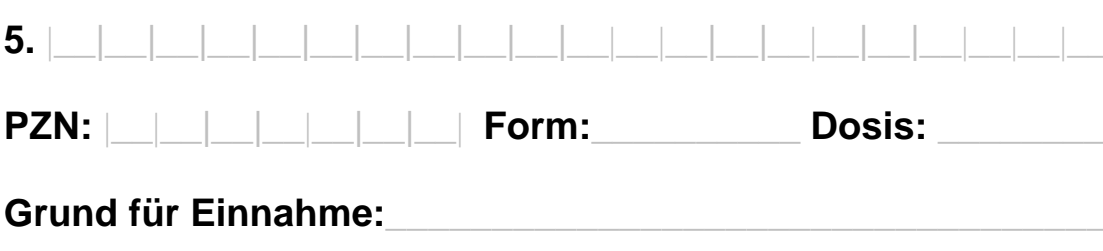 & $\begin{array}{l}\square \mathrm{N} 1 \\
\square \mathrm{N} 2 \\
\square \mathrm{N3}\end{array}$ & $\square$ & $\square$ & $\square$ & $\square$ \\
\hline 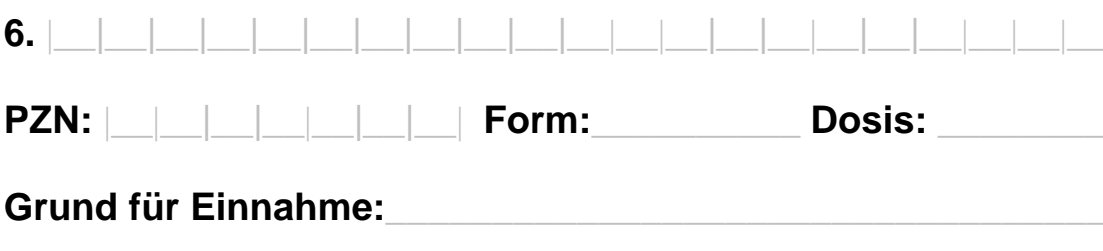 & $\begin{array}{l}\square \mathrm{N} 1 \\
\square \mathrm{N} 2 \\
\square \mathrm{N} 3\end{array}$ & $\square$ & $\square$ & $\square$ & $\square$ \\
\hline 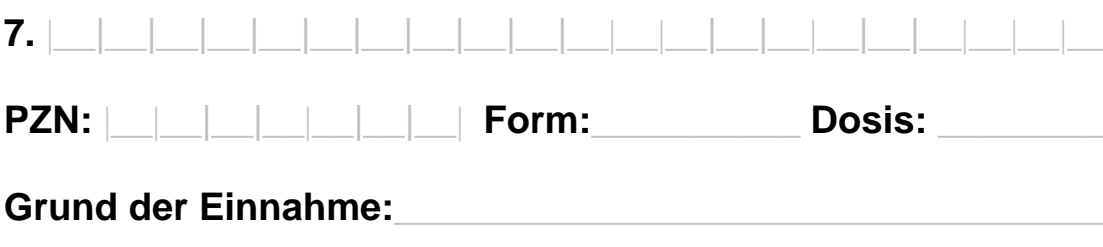 & $\begin{array}{l}\square \mathrm{N} 1 \\
\square \mathrm{N} 2 \\
\square \mathrm{N} 3\end{array}$ & $\square$ & $\square$ & $\square$ & $\square$ \\
\hline 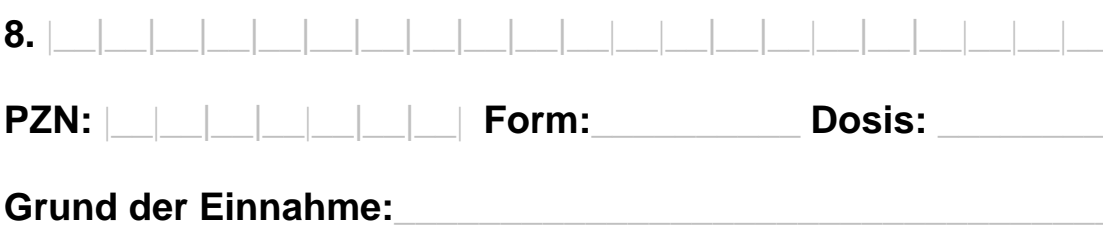 & $\begin{array}{l}\square \mathrm{N} 1 \\
\square \mathrm{N} 2 \\
\square \mathrm{N} 3\end{array}$ & $\square$ & $\square$ & $\square$ & $\square$ \\
\hline
\end{tabular}


Die folgenden Fragen beziehen sich auf Ihren Blutdruck.

1. Seit wann wissen Sie, dass Ihr Blutdruck zu hoch ist?

$\square_{1}$ Seit weniger als 6 Monaten.

$\square_{2}$ Seit 6-12 Monaten.

$\square_{3}$ Seit 1-2 Jahren.

$\square_{4}$ Seit 2-5 Jahren.

$\square_{5}$ Seit 5-10 Jahren.

$\square_{6}$ Seit mehr als 10 Jahren.

$\square_{7}$ Ich bin mir nicht sicher.

2. Seit wann erhalten Sie Medikamente gegen den hohen Blutdruck?

$\square_{1}$ Seit weniger als 6 Monaten.

$\square_{2}$ Seit 6-12 Monaten.

$\square_{3}$ Seit 1-2 Jahren.

$\square_{4}$ Seit 2-5 Jahren.

$\square_{5}$ Seit 5-10 Jahren.

$\square_{6}$ Seit mehr als 10 Jahren.

$\square_{7}$ Ich erhalte keine Medikamente gegen hohen Blutdruck.

3. Wer hat Ihren Blutdruck zuletzt gemessen?

$\square_{1}$ Meine Hausärztin/mein Hausarzt.

$\square_{2}$ Die Apothekerin/der Apotheker.

$\square_{3}$ Ich messe meinen Blutdruck selbst.

$\square_{4}$ Eine andere Person, und zwar

$\square_{5}$ Ich kann mich nicht erinnern.

4. Wann wurde Ihr Blutdruck das letzte Mal gemessen?

$\square_{1}$ Innerhalb der letzten 7 Tage.

$\square_{2}$ Vor 1-4 Wochen.

$\square_{3}$ Vor 1-3 Monaten.

$\square_{4}$ Vor mehr als 3 Monaten.

$\square_{5}$ Ich kann mich nicht erinnern.

5. Wie hoch war Ihr Blutdruck bei der letzten Messung?

Ich kann mich nicht erinnern. $\mathrm{mm} \mathrm{Hg}$ (Z.B. 150/90).

6. Erinnern Sie sich noch, ob Ihr oberer Blutdruckwert in der Zeit vor der Einnahme von Blutdruckmedikamenten über oder unter $160 \mathrm{~mm} \mathrm{Hg}$ betrug?

$\square_{1} \mathrm{Ja}$, ich kann mich erinnern:

$\square_{11}$ Der obere Wert betrug über $160 \mathrm{~mm} \mathrm{Hg}$ (z.B. 180/100).

$\square_{12}$ Der obere Wert betrug unter $160 \mathrm{~mm} \mathrm{Hg}$ (z.B. 150/90).

$\square_{2}$ Nein, ich kann mich nicht erinnern.

$\square_{3}$ Ich habe noch nie Medikamente gegen Bluthochdruck genommen. 
Bei den folgenden Fragen geht es um die Einnahme von Blutdruckmedikamenten und um Gewohnheiten oder Probleme, die dabei eine Rolle spielen könnten.

\section{gar
nicht selten $\begin{gathered}\text { mei- } \\ \text { stens }\end{gathered}$ immer}

1. Wie oft vergessen Sie, Ihre Blutdruckmedikamente einzunehmen?

2. Wie oft entscheiden Sie sich, Ihre Blutdruckmedikamente nicht einzunehmen?

3. Wie oft essen Sie salziges Essen?

4. Wie oft salzen Sie Ihre Mahlzeiten nach?

$\begin{array}{lll}\square_{1} & \square_{2} & \square_{3}\end{array}$

Wie oft essen Sie Fertigprodukte oder Fast Food

5. (z.B. Tüten- oder Dosensuppen, fertige Tiefkühlgerichte, Pizza, Hamburger, Currywurst)?

$\begin{array}{lll}\square_{1} & \square_{2} & \square_{3}\end{array}$ $\square_{4}$

6. Wie oft vereinbaren Sie nach einem Besuch bei Ihrer Hausärztin/Ihrem Hausarzt einen Kontrolltermin?

7. Wie oft versäumen Sie einen vereinbarten Termin bei

7. Ihrer Hausärztin/Ihrem Hausarzt?

8. Wie oft vergessen Sie, sich ein neues Rezept für Ihr

8. Blutdruckmedikament zu bestellen?

9. Wie oft gehen Ihnen Ihre Blutdruckmedikamente aus, ohne dass Sie ein neues Rezept haben?

10. Wie oft unterlassen Sie die Einnahme Ihrer

Blutdruckmedikamente vor einem Arzttermin?

11. Wie oft versäumen Sie die Einnahme Ihrer

Blutdruckmedikamente, wenn Sie sich gut fühlen?

$\square_{1}$

$\square_{2}$

$\square_{3}$

$\square_{4}$

12. Wie oft versäumen Sie die Einnahme Ihrer

12. Blutdruckmedikamente, wenn Sie sich krank fühlen?

Wie oft nehmen Sie die Blutdruckmedikamente

13. anderer Personen ein (z.B. von Verwandten, Freunden)?

14. Wie oft sind Sie nachlässig und versäumen die
Einnahme Ihrer Blutdruckmedikamente? 


\section{Die folgenden Fragen sind wichtig für die Beschreibung} der Gruppe der Studienteilnehmer/innen.

\section{Sind Sie}
$\square_{1}$ weiblich?
$\square_{2}$ männlich?

2. Welche Staatsangehörigkeit haben Sie?
$\square_{1}$ deutsch
$\square_{2}$ nicht deutsch

3. Wann sind Sie geboren?

Geburtsmonat:

Geburtsjahr:

4. Leben Sie mit einer Partnerin/einem Partner zusammen?
$\square_{1} \mathrm{ja}$
$\square_{2}$ nein

\section{Welchen Familienstand haben Sie?}
$\square_{1}$ Ich bin verheiratet und lebe mit meiner Ehepartnerin/meinem Ehepartner zusammen.
$\square_{2}$ Ich bin verheiratet und lebe nur am Wochenende mit meiner Ehepartnerin/ meinem Ehepartner zusammen.
$\square_{3}$ Ich bin verheiratet und lebe von meiner Ehepartnerin/meinem Ehepartner getrennt.
$\square_{4}$ Ich bin ledig.
$\square_{5}$ Ich bin geschieden.
$\square_{6}$ Ich bin verwitwet.

6. Wie viele Personen leben ständig in Ihrem Haushalt?
$\square_{1}$ Ich lebe mit Person/en zusammen, davon ist/sind unter 18 Jahre alt.
$\square_{2}$ Ich lebe allein.

\section{Welchen höchsten allgemein bildenden Schulabschluss haben Sie?}

$\square_{1}$ Ich bin noch Schüler/in.

$\square_{2}$ Ich bin ohne Hauptschulabschluss (Volksschulabschluss) von der Schule abgegangen.

$\square_{3}$ Ich habe den Hauptschulabschluss (Volksschulabschluss).

$\square_{4}$ Ich habe den Realschulabschluss (Mittlere Reife).

$\square_{5}$ Ich habe den Abschluss der Polytechnischen Oberschule, 10. Klasse (vor 1965: 8. Klasse).

$\square_{6}$ Ich habe die Fachhochschulreife.

$\square_{7}$ Ich habe die allgemeine oder fachgebundene Hochschulreife/Abitur (Gymnasium bzw. EOS).

$\square_{8}$ Ich habe einen anderen Schulabschluss, und zwar: 


\section{Welchen beruflichen Ausbildungsabschluss haben Sie?}

Was trifft auf dieser Liste auf Sie zu? Bitte lesen Sie die Liste ganz durch; Mehrfachnennungen sind möglich.

$\square_{1}$ Ich bin in beruflicher Ausbildung (Auszubildende/r, Student/in).

$\square_{2}$ Ich habe eine beruflich-betriebliche Berufsausbildung (Lehre) abgeschlossen.

$\square_{3}$ Ich habe eine Ausbildung an einer Fach-, Meister-, Technikerschule, Berufsoder Fachakademie abgeschlossen.

$\square_{4}$ Ich habe eine beruflich-schulische Ausbildung (Berufsfachschule, Handelsschule) abgeschlossen.

$\square_{5}$ Ich habe einen Fachhochschulabschluss.

$\square_{6}$ Ich habe einen Hochschulabschluss.

$\square_{7}$ Ich habe einen anderen beruflichen Abschluss, und zwar:

$\square_{8}$ Ich habe keinen beruflichen Abschluss und bin nicht in beruflicher Ausbildung.

\section{Sind Sie zurzeit erwerbstätig? Was auf dieser Liste trifft auf Sie zu?}

Unter Erwerbstätigkeit wird jede bezahlte bzw. mit einem Einkommen verbundene Tätigkeit verstanden, egal welchen zeitlichen Umfang sie hat.

$\square_{1}$ Ich bin zurzeit nicht erwerbstätig (Student/in, Arbeitssuchende/r, Null-Kurzarbeiter/in, im Vorruhestand, Rentner/in). (Bitte weiter mit Frage 10!)

$\square_{2}$ Ich bin in Vollzeit erwerbstätig mit einer wöchentlichen Arbeitszeit von 35 Stunden und mehr.

$\square_{3}$ Ich bin in Teilzeit erwerbstätig mit einer wöchentlichen Arbeitszeit von 15 bis 34 Stunden. (Bitte weiter mit Frage 10!)

$\square_{4}$ Ich bin in Teilzeit oder stundenweise erwerbstätig mit einer wöchentlichen Arbeitszeit unter 15 Stunden. (Bitte weiter mit Frage 10!)

$\square_{5}$ Ich habe einen „Ein-Euro-Job“ (und beziehe Arbeitslosengeld 2 nach Harz IV).

$\square_{6}$ Ich bin in Mutterschaftsurlaub, Elternzeit oder in sonstiger Beurlaubung.

$\square_{7}$ Ich bin Auszubildende/r, Lehrling, Umschüler/in.

10. Nur ausfüllen, wenn Sie nicht in Vollzeit erwerbstätig sind!

Bitte kreuzen Sie an, zu welcher Gruppe auf dieser Liste Sie gehören:

$\square_{1}$ Ich bin Student/in.

$\square_{2}$ Ich bin Rentner/in, Pensionär/in, im Vorruhestand.

$\square_{3}$ Ich bin zurzeit arbeitssuchend oder mache Kurzarbeit.

$\square_{4}$ Ich bin Hausfrau/Hausmann.

$\square_{5}$ Ich bin aus anderen Gründen nicht in Vollzeit erwerbstätig, und zwar: 
11. Welche berufliche Stellung trifft derzeit auf Sie zu bzw. welche berufliche Stellung hatten Sie zuletzt bei Ihrer früheren Erwerbstätigkeit?

$\square_{1}$ Ich bin/war Akademiker/in in Selbständigkeit oder in einem freien Beruf (Ärztin/ Arzt, Rechtsanwältin/Rechtsanwalt, Steuerberater/in oder ähnliches).

$\square_{2}$ Ich bin/war selbständig im Handel, Gewerbe, Handwerk, Industrie, Dienstleistung, auch Ich-AG oder PGH-Mitglied.

$\square_{3}$ Ich bin/war Landwirt/in bzw. Genossenschaftsbauer oder Genossenschaftsbäuerin in Selbständigkeit.

$\square_{4}$ Ich bin/war Beamtin/Beamter, Richter/in, Berufssoldat/in.

$\square_{5}$ Ich bin/war Angestellte/r.

$\square_{6}$ Ich bin/war Arbeiter/in.

$\square_{7}$ Ich bin/war in beruflicher Ausbildung.

$\square_{8}$ Ich bin/war mithelfende/r Familienangehörige/r.

$\square_{9}$ Ich bin/war aus anderen Gründen nicht erwerbstätig, und zwar:

12. Wie hoch ist das monatliche Nettoeinkommen Ihres Haushalts insgesamt?

Gemeint ist dabei die Summe, die sich aus Lohn, Gehalt, Einkommen, aus selbständiger Tätigkeit, Rente oder Pension jeweils nach Abzug der Steuern und Sozialversicherungsbeiträge ergibt. Rechnen Sie bitte auch die Einkünfte aus öffentlichen Beihilfen, Einkommen aus Vermietung, Verpachtung, Wohngeld, Kindergeld und sonstige Einkünfte hinzu.

$\square_{1}$ unter $500 €$

$\square_{2} 500$ bis unter $1000 €$

$\square_{3} 1000$ bis unter $1500 €$

$\square_{4} 1500$ bis unter $2000 €$

$\square_{5} 2000$ bis unter $2500 €$

$\square_{6} 2500$ bis unter $3000 €$

$\square_{7} 3000$ bis unter $3500 €$

$\square_{8} 3500 €$ und mehr

\section{Bitte überprüfen Sie noch einmal, ob Sie alle Fragen beantwortet haben.}




\section{Danksagung}

Ich danke Herrn Prof. Dr. Kochen, MPH, FRCGP für die freundliche Überlassung des Themas der Dissertation.

Mein besonderer Dank gilt Frau Dr. Janka Koschack für die ausgezeichnete Betreuung während der Arbeit. Durch ihre in hohem Maße engagierte und gleichzeitig herzliche Art war die Zusammenarbeit sehr erquicklich. Eine bessere Betreuung hätte ich mir nicht wünschen können.

Schließlich danke ich den Mitarbeiterinnen der Abteilung Allgemeinmedizin, Frau Gabriella Marx und Frau Hannelore Schneider-Rudt, für die Unterstützung bei der Datenerhebung. 


\section{Lebenslauf}

Am 09. Juli 1979 wurde ich unter dem Namen Jörg Schnakenberg in Bremen geboren. Mit sechs Jahren zogen meine Eltern Elke und Jürgen Schnakenberg mit meinem zwei Jahre jüngeren Bruder Stefan und mir in die Gemeinde Grasberg im Umland von Bremen. Dort besuchte ich Kindergarten und Grundschule. Die Orientierungsstufe absolvierte ich in Worpswede, das Gymnasium besuchte ich bis zum Abitur 1998 in Lilienthal. Anschließend begann ich im Oktober desselben Jahres ein Studium der Produktionstechnik in Bremen. Im Jahr 2000 wurde ich schließlich zum Zivildienst eingezogen, den ich in einer Dialysegemeinschaftspraxis in Freiburg im Breisgau ableistete. Durch die dort tätigen Ärzte wurde ich für die Medizin begeistert und zog erstmals ein Medizinstudium in Erwägung. Nach dem Zivildienst immatrikulierte ich mich im WS 2001/2002 nochmals für einen Ingenieurstudiengang an der TU IImenau. Mein Enthusiasmus für dieses Fach war allerdings nach wie vor begrenzt, so dass ich schließlich im SS 2002 das Medizinstudium in Göttingen aufnahm. Im Frühjahr 2004 legte ich die ärztliche Vorprüfung mit der Note sehr gut ab. Von 2005 bis 2007 war ich als studentische Hilfskraft im Kurs für Medizinische Basisfähigkeiten der Abteilung Allgemeinmedizin tätig. Mein Praktisches Jahr verbrachte ich von 2007 bis 2008 im Klinikum links der Weser in Bremen, sowie im Kantonsspital Uri in Altdorf (Schweiz). Das Medizinstudium schloss ich am 26. November 2008 mit der Note gut ab. Die Approbation als Arzt wurde mir am 09. Dezember 2008 erteilt. 